\title{
More than superstition: Differential effects of featural heterogeneity and change on subitizing and counting
}

\author{
LANa M. Trick \\ University of Guelph, Guelph, Ontario, Canada
}

\begin{abstract}
This study investigates the effects of item heterogeneity (differences in color and shape) and moment-to-moment feature change as it relates to the issue of whether subitizing and counting involve different processes. Participants enumerated displays of up to eight items that were either homogeneous or heterogeneous. In situations where the heterogeneous displays always had approximately half of the items of one type and half of the other, heterogeneity significantly sped enumeration in the counting range (6-8 items) and significantly slowed enumeration in the subitizing range (1-3 items), a dissociation that suggests that subitizing and counting involve different operations. Moment-to-moment feature change had no effect on subitizing. However, feature change slowed counting, but only when participants were enumerating heterogeneous items that were half of one type and half of the other, as might be expected if participants were using differences in features to select items by type.
\end{abstract}

It has long been known that when people enumerate visual-spatial items, such as dots laid out in different locations all at one time, and they are instructed to indicate the number of items as fast and as accurately as they can, there are different patterns of performance with fewer than four or five dots than with more (e.g., Jevons, 1871). With small numbers of dots, enumeration is effortless, accurate, and fast, but with larger numbers, enumeration is effortful, relatively error-prone, and slow: For example, response time (RT) slope $=40-100 \mathrm{msec} /$ item for 1-3 items as opposed to $200-350 \mathrm{msec} /$ item for more. Subitizing is the name given to the rapid, effortless form of enumeration that occurs for small numbers of dots, and the subitizing range is the range of numbers over which subitizing is observed (Kaufman, Lord, Reese, \& Volkmann, 1949). There are individual differences in the maximal number of items that can be subitized, but for most adults the subitizing range is between 1-3 and 1-5 items (Akin \& Chase, 1978; Jensen, Reese, \& Reese, 1950; Mandler \& Shebo, 1982; Trick \& Pylyshyn, 1993). Counting is the name given to the more laborious and time consuming form of enumeration used with larger numbers of dots; the counting range is the range of numerosities over which counting is observed. There has been controversy about whether subitizing and counting are different processes or the same process applied to different numbers of items; in fact, some have gone so far as to say that subitizing is "mere superstition" and not a real phenomenon (Balakrishnan \& Ashby, 1992; Cordes \& Gelman, 2005). However, if there is no such thing as subitizing, if the enumeration of small and large numbers is accomplished by exactly the same mechanism, there would be no reason to expect a manipulation to have opposite effects on enumeration in the subitizing and counting ranges. These studies investigate the effects of featural heterogeneity and change on the enumeration of 1-8 items as it is influenced by attentional selection. The results have ramifications for enumeration literature and the research on selective attention.

The debate about subitizing and counting is complicated by the fact that there are not just two proposed enumeration processes - but three. For example, Smitsman (1982) discusses two types of enumeration that calculate precise number (subitizing and counting), and a third that delivers approximate number (estimation). The empirical markers that distinguish among the three types of enumeration involve a combination of processing speed and accuracy. Subitizing and estimation are fast, although the increase in the amount of time for each additional item (the RT slope) is different for subitizing and estimation, and subitizing is extremely accurate, whereas estimation is extremely inaccurate (the exact number is typically wrong, though the estimate may be in the general vicinity). Similarly, both subitizing and counting are accurate (though subitizing is more accurate) but the RT slope is much smaller for subitizing than for counting. To further complicate matters, in any specific experiment there may be a variety of variables that could serve as heuristics to number, given that participants typically see several hundred trials with the same items. For example, they may begin to use cues such as contour density and spatial extent (the more items, the larger total area covered by items). Furthermore, when the same displays are shown again and again, participants may even use item configuration to infer number, as occurs, for

L.M.Trick, Itrick@uoguelph.ca 
example, when participants read dice (Mandler \& Shebo, 1982). Consequently, it is not always clear whether participants are responding to number per se or to a variable that is confounded with number in a particular experiment.

The contention that subitizing and counting require different processes rests primarily on data that show they have different RT slopes. However, Balakrishnan and Ashby (1992) found that a bilinear function was a poor fit to some enumeration data they had collected; consequently, they concluded that there was no such thing as subitizing. Methodological problems made their enumeration data difficult to interpret, though. They had 4 participants enumerate 1-8 square dots over ten 2 -h sessions. Items were always presented in a horizontal line in which there were 10 possible item positions (or, for 2 participants, 14) and those positions were evenly spaced. Moreover, each item had a different color. As a result, item density, regularity of item spacing, and the amount of color and brightness heterogeneity in the image were all potential cues to numerosity. Furthermore, the study used 200-msec limited-duration displays. It was Jensen, Reese, and Reese (1950) who first pointed out that it is a bad idea to limit exposure duration if the goal is to study subitizing and counting in the same experiment, because this procedure may actually change the nature of the processes under investigation. Counting is a reasonably accurate process that delivers precise number. In this study, the error rates were very high for larger numbers of items (some approaching 60\%), as might be expected if participants were using some combination of estimation, heuristics, and guesswork, rather than actually counting. Limited exposure durations prevent the eye movements that are necessary if participants are to see the items, let alone enumerate them. These eye movements are especially important when there are larger numbers of items because, as the number of items increases, on average they become more densely packed and occupy larger total areas of space; the spatial resolution of sensory and/or attentional processes is worse in the periphery (Intriligator $\&$ Cavanagh, 2001). Eye movements inflate RT slopes in both the subitizing and counting ranges, but there is ample evidence that eye movements do not cause subitizing and counting from studies that control eye movements in other ways (Klahr \& Wallace, 1976; Watson, Maylor, \& Bruce, 2005a; see Table 1 in Trick \& Pylyshyn, 1994, for a presentation of subitizing and counting slopes from enumeration studies). There is little to be gained and much to be lost by limiting display duration.

Nonetheless, even had the methodology been unassailable and the predicted deviations from linearity emerged, it would still be unclear whether the function derived from one process applied to different numbers of items or whether it derived from different processes applied to different numbers of items. RT and error data are insufficient in themselves to distinguish one process from two. It would be better to look for supporting evidence in dissociations: cases where a manipulation has different (preferably opposite) effects on subitizing and counting, or, more properly, on enumeration when subitizing and counting are evident from differences in RT slope. If subitizing and counting are the same process, it is hard to explain how a manipulation impedes enumeration in one range and facilitates it in another.
When looking for a variable that might produce such a dissociation, a promising place to start is by examining the effects of differences in item features (heterogeneity in color and shape): the types of difference that prompt effortless texture segmentation and efficient search (Beck 1982; Julesz, 1984; Treisman \& Gelade, 1980; Wolfe, 1994). These are interesting because there is evidence that participants can perform selective enumeration (enumerating selected targets among distractors) when targets and distractors differ in terms of basic features. Moreover, under these circumstances, the deviations in RT slope indicative of subitizing and counting are evident-but only if the targets and distractors differ in terms of their features (Trick \& Pylyshyn, 1993, 1994).

The only theory that predicts this pattern of results is one that incorporates enumeration into a more general theory of vision and visual attention (Trick \& Pylyshyn, 1994). According to this account, subitizing and counting are phenomena specific to spatial enumeration - the enumeration of objects laid out in different locations in space at one time. This is not to deny that other forms of enumeration exist, but rather to suggest that the deviations from linearity in RT slope indicative of the change from subitizing and counting have been shown only in spatial enumeration, and only in some forms of spatial enumeration at that.

Trick and Pylyshyn (1994) proposed that the RT slope differences between subitizing and counting emerge because of limitations in the mechanism that individuates small numbers of selected objects in multielement spatial displays by assigning mental reference tokens called FINSTs (fingers of instantiation). This system is more generally used for visual-motor coordination and deriving spatial relations between parts of an item. Once the basic features in the image have been derived (basic color and shape), FINSTs can be assigned selectively to items with one type of feature or another ("activated areas on a master map of item saliencies," to use the terminology of the visual search literature). However, this is possible only if targets and distractors differ in terms of properties that can be derived in parallel across the display by early vision - properties such as color and shape. If distinguishing between targets and distractors requires the one-area-at-a-time type of processing seen in operations that employ the attentional focus, the rapid accurate subitizing process cannot be used for enumeration, even if there are only 1-3 items. Instead, the slow, laborious counting process is used; as a result, there is no longer a difference in RT slopes between 1-3 and 6 or more items.

According to this account, subitizing occurs when there are fewer items than there are mental reference tokens (FINSTs). Reference tokens are assigned to each item, and the number name can then be acquired from semantic memory. Basically, the latter stage can be understood as a type of categorization, because it involves choosing a number name to match a selected set of elements within the scene, as occurs when participants assign a name (e.g., cat) to a selected grouping of contours (a proto-object). However, number categorization differs from other forms of categorization insofar as displays of higher numerosity contain displays of lower numerosity. For example, within every 3-item display, there are 2 -item and 1-item displays. This would be analogous to 
a situation in which there were birds and butterflies within every image of a cat. The only way to determine numerosity is to find either the highest number supported by the display (the number at which every assigned FINST is matched by a number name) or the lowest number not supported by the display minus one - and the latter is unlikely, given that it is difficult to look for the absence of features. Thus, one way to choose the appropriate numeric response is to match each selected item to a number name, in the order of the number names. At least some of the RT slope in the subitizing range seems to originate from this response choice-matching process, because studies that exclude response choice from timing have smaller increases in RT with each additional item (see Trick \& Pylyshyn, 1994, Table 1).

The "counting" process is more complex. It is unfortunate that Kaufman, Lord, Reese, and Volkmann (1949) chose the word counting to refer to the accurate timeconsuming process used to enumerate larger numbers of dots, because, in common usage, counting refers to enumeration in general. In fact, counting is sometimes used to refer to the process of listing the number names, one at a time, in order, even when there is nothing to count. Confusion about the meaning of the word counting has produced unnecessary controversy. Furthermore, in dot displays, where the differences in RT slope indicative of subitizing and "counting" are most evident, adults report using a group-and-add strategy rather than a one-by-one enumeration (e.g., Klahr \& Wallace, 1976; van Oeffelen \& Vos, 1982). According to Trick and Pylyshyn (1994), the reason that group-and-add counting occurs is that the FINST reference tokens have to be reused when there are more than 3 or 4 items. Reference tokens are repeatedly assigned, first to one group of items and then to the next, until all of the items have been individuated, and this requires a variety of operations beyond those used in subitizing.

Specifically, group-and-add counting requires grouping items, selecting a specific group of items to start with (directing the attentional focus to that group), subitizing items within the group, putting the resultant number into a running total in working memory, marking the items as already attended so that items are not enumerated again, selecting the next group of items, subitizing the items within that group, adding that new number of items into the running total, and continuing until all the groups have been marked off as enumerated. Group-and-add counting involves many operations, and it is more error-prone than subitizing because there are more things to go wrong: addition errors and memory lapses that cause people to forget the running total or the location of already-enumerated items. Although some of the operations in counting might take place in parallel (e.g., grouping processes), the RT slope is much higher in counting than in subitizing, because in counting, the more items there are the more times the attentional focus has to be moved from group to group, the more times subitizing has to occur, the more additions have to be performed, and so on.

Given this account, it is interesting to consider the impact of featural heterogeneity as it relates to attentional selection. Selection involves choosing some things to process and not others, and it is almost always necessary in enumeration because people typically do not enumerate every contour in the visual field. (In a typical dot enumeration study, participants enumerate the dots and not the edges of the computer monitor, for example.) Nonetheless, selection is crucial in the counting range because there are too few FINSTs to individuate every item that has to be enumerated at once. As a result, participants select one group to individuate, then the next, until every group has been enumerated. When items are homogeneous, the basis for selection is necessarily spatial: A person might choose to start with groups on the left and work right, the groups on the top and work down, and so on. However, when items are heterogeneous, and heterogeneous in such a way that early vision could use heterogeneity to define different groups (as in grouping by similarity), an individual might form a strategy of selecting items by feature. For example, a person might select the red items first, then the blue.

Selection is typically under top-down control in enumeration; consequently, it can be influenced by expectations and strategies. Six studies are described in which contextual factors are manipulated to encourage participants to adopt or abandon an attentional set to select items by type when enumerating heterogeneous displays. The first three show that heterogeneity can have different effects, depending on whether participants expect the items to be approximately half of one type and half of another (this determines whether they form an attentional set to select items by type). This attentional set has opposite effects on performance in the subitizing and counting ranges. The last three studies are about how featural change influences this attentional set to select items by type.

\section{EXPERIMENT 1 \\ Enumerating Heterogeneous Items With an Attentional Set to Select Items by Type}

In these studies, the goal was to induce an attentional set to select items by type when enumerating displays of heterogeneous items. There are several ways to encourage participants to adopt an attentional set, the most obvious of which is simply to tell them what to do. However, this practice introduces experimental demand. Instead, attentional set was allowed to develop naturally with repeated presentations of heterogeneous displays in which there was a regularity that could be exploited to speed enumeration. Typically, there is a lot of trial-to-trial uncertainty in group-and-add counting, because with random configurations of items the number of groups and the number within a group vary on the basis of grouping by proximity for any given number of items (van Oeffelen \& Vos, 1982, 1983, 1984). For example, a 7-item display might involve a group of 5 plus a group of 2 ; a group of 4 plus a group of 3 ; or even three groups $(2+2+3)$; and so on. This uncertainty is unavoidable when items are homogeneous and randomly distributed. However, if every display of heterogeneous items had approximately equal numbers of each type of item, the addition would always be the same for a given number of items; moreover, it would be an addition where the addends were approximately equal. For example, 7-item displays would always involve a group of 4 and a group of 3 (e.g., either 4 red and 3 blue items, or 4 blue and 3 red items). This would speed the addition opera- 
tion in group-and-add counting, because latencies decrease with repeated presentations of the same addition problem.

The effect of display (homogeneous, heterogeneous) was measured as a function of the number of items. It was predicted that subitizing and counting would always be evident, which is to say that the usual differences in RT slope between small and large numbers of items would occur in every condition of every experiment. Although some manipulations make subitizing impossible (Trick \& Pylyshyn, 1993), the manipulations in these studies should not, because they do not compromise unit formation or interfere with the system that individuates spatial items by assigning reference tokens.

The second prediction was that there would be a crossover interaction between display type and number of items, with heterogeneity speeding enumeration in the counting range and slowing it in the subitizing range. In particular, when participants are led to expect that approximately half the items in heterogeneous displays will be of one type and half of the other type, heterogeneity should speed enumeration in the counting range, because it should speed the addition operation in group-and-add counting. RT should be lower for heterogeneous than for homogeneous displays. No display $X$ number interaction was expected within the counting range because the display manipulation facilitates addition, and in most cases there should only be a single addition operation when there are only up to 8 items. Van Oeffelen and Vos $(1982,1983,1984)$ found that, when participants group homogeneous items by relative proximity for group-and-add counting, they are biased both to minimize the number of groups and ensure a subitizable number within each group. Given these constraints, it seems likely that there may be the occasional three-group (two addition) display for 7 or 8 items, but these should be infrequent. Similarly, although addend size affects addition latencies (Parkman \& Groen, 1971), within the range of numerosities in these studies the differences in addend size between homogeneous and heterogeneous displays should not be so large as to produce a display $\times$ number interaction. Within this limited range, it is primarily the reduction in uncertainty that produces the effect.

In contrast, display heterogeneity should slow enumeration in the subitizing range. Subitizing does not require addition, so the heterogeneity manipulation that speeds addition in group-and-add counting would not benefit subitizing. Furthermore, selecting items by type would only serve to slow things down when there are more than enough FINSTs to enumerate every item at once. When participants form an attentional set to select items by type, heterogeneity should slow enumeration in the subitizing range, though RT slopes should be relatively unaffected, because the process of releasing the restriction (opening selection up to both kinds of item) would only have to occur once, not once per item. It was further predicted that when the display type manipulation was blocked so that all the heterogeneous displays were presented in a group (Experiment 1A), heterogeneity would even slow the enumeration of one item, though there can be no heterogeneity in a display with a single item. It is the expectation for heterogeneity, rather than heterogeneity per se, that produces the effect.

\section{Method}

Participants. There were 24 participants ( 21 females), 12 in each experiment. All were university students 19-21 years of age, with normal color vision and normal or corrected-to-normal acuity. They were paid in course credit.

Apparatus and Stimuli. Testing was conducted on a Macintosh G4 computer. Displays were made up of 1-9 colored shapes that were randomly positioned on a $7 \times 7$ notional grid on the computer screen. The grid occupied an $11.8^{\circ} \times 12.1^{\circ}$ visual angle area when viewed from $45 \mathrm{~cm}$. Shapes were presented on a white background [RGB $(255,255,255), 32 \mathrm{~cd} / \mathrm{m}^{2}$ ] with $0.88^{\circ}$ visual angle the minimum distance between shapes. Because later experiments measured the effects of featural change on one as opposed to two dimensions, a strong heterogeneity manipulation was used: Heterogeneous items differed in terms of both color and shape. The shapes were $1.02^{\circ} \times$ $1.02^{\circ}$ red squares [RGB $\left.(255,0,0), 12 \mathrm{~cd} / \mathrm{m}^{2}\right]$ and blue triangles [RGB $\left.(0,0,255), 12 \mathrm{~cd} / \mathrm{m}^{2}\right]$.

For both experiments, in the homogeneous condition all items in a given display were identical; displays of red squares and blue triangles were presented in a random order. In the heterogeneous condition, displays had both red squares and blue triangles. The number of red squares and blue triangles in a given display was exactly equal for even numbers of items, and approximately equal for odd numbers of items (with one more of one type than of the other). When there were unequal numbers of items, half the time there were more red squares and half the time there were more blue triangles. Positions for different item types were randomized so that items did not necessarily fall into homogeneous groups, although this could happen. Each display was unique for each participant. Sample displays are shown in Figure 1.

Procedure. In all of the experiments, participants were told that their task was to enumerate colored shapes as fast as they could, with accuracy. Item heterogeneity was never explicitly mentioned in the instructions. The sequence of events was the same for all experiments. At the beginning of the trial, a $1.02^{\circ} \times 1.02^{\circ}$ visual angle fixation cross was presented in the center of the screen for $455 \mathrm{msec}$. Next, the enumeration display was presented and remained until participants hit the space bar to indicate that they knew how many items there were (the timed portion of the trial). At that point, the enumeration display disappeared and participants typed in the number of items. Error feedback was given after each trial in the form of a " + " if the answer was correct, or a "-" if it was not. Feedback remained onscreen for $455 \mathrm{msec}$. This two-phase paradigm has been used with success in other studies (e.g., Trick \& Enns, 1997a; Watson \& Maylor, 2006).

Each of the participants did 288 experimental trials ( 8 blocks of 36 ). In Experiment 1A, display heterogeneity was blocked and the order counterbalanced; in Experiment 1B, this manipulation was randomized. For both experiments, the total number of practice trials was 36 .

\section{Results and Discussion}

The same data analysis strategy was used in all of the experiments described in this article. First, data from the top number in the number range ( 9 for these studies) were excluded because of end effects, which occur when participants infer the maximal number of items $(n)$ that can appear in displays, and form the strategy of guessing $n$ whenever there are large numbers of items (Mandler \& Shebo, 1982; Trick \& Pylyshyn, 1994). End effects produce disproportionately low RTs for correct trials when there are $n$ items and exaggerated error rates at $n-1$. The practice of dropping the largest number in the range is common in enumeration studies (Trick \& Pylyshyn, 1993; Watson \& Maylor, 2006).

Second, because RT analyses were performed on data from trials in which the response was accurate, data from participants with error rates in excess of $30 \%$ for any specific condition and number were not included; in these instances, there were too few RTs to analyze. Also, analyzing 
latencies from individuals with extremely high error rates is inappropriate, because these studies are about subitizing and counting (relatively accurate processes) rather than estimation. None of the participants in Experiments $1 \mathrm{~A}$ and $1 \mathrm{~B}$ had error rates in excess of $30 \%$ for any number or condition.

Third, RT data were screened for outliers. Typically, in RT studies the experimental software times out after a short period to avoid inclusion of extreme latencies in the means. Given that it takes so much longer to enumerate 9 items than to enumerate 1, this was not feasible. Instead, for each participant, RTs were dropped if they fell outside two standard deviations of the mean for that specific individual for that specific condition and number of items. This resulted in the loss of $5.2 \%$ and $4.7 \%$ of the trials for Experiments 1A and 1B, respectively.

Fourth, error analyses were carried out. Overall, error rates were low $(M=3.4 \%$ and $2.4 \%$, for Experiments $1 \mathrm{~A}$ and $1 \mathrm{~B}$, respectively), as might be expected if participants followed the directions to enumerate with accuracy. As always, error rates increased with the number of items [Experiment 1A, $F(7,77)=4.19, p=.001, \eta^{2}=.28, M$ error $=1.0 \%-6.5 \%$; Experiment $1 \mathrm{~B}, F(3.10,34.08)=$ $5.74, p=.003, \eta^{2}=.34, M$ error $\left.=0.0 \%-4.7 \%\right]$. Error analyses revealed no main effects or interactions involving display type in either experiment ( $p>.1$ for all).

The final stages involved RT analyses. RT calculations began with trend analysis and regression to determine whether there was evidence of subitizing and counting (deviations from linearity in the enumeration function and significant differences in RT slope between small and large numbers of items). ANOVAs were then performed; if the sphericity assumption was violated, the GreenhouseGeisser correction was applied to the degrees of freedom (Kirk, 1982, p. 83). Finally, if a condition $\times$ number interaction emerged, separate analyses were carried out within the subitizing and counting ranges. Because there are individual differences in the maximal number of items that can be subitized, and to avoid mixing data from participants

\section{Experiments $1 \mathrm{~A}$ and $1 \mathrm{~B}$ (Attentional Set)}

Homogeneous Condition

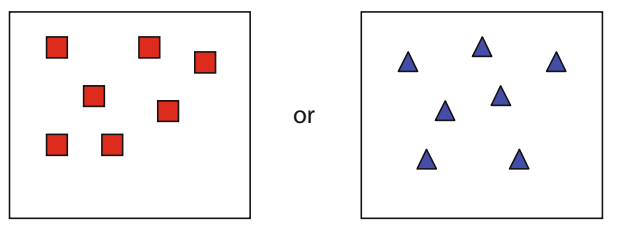

Heterogeneous Condition

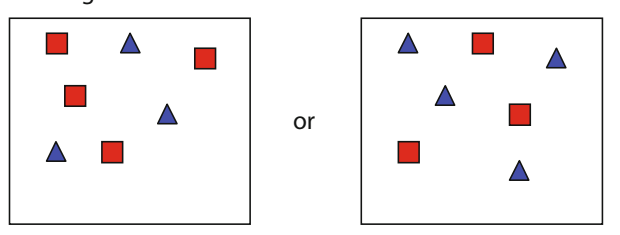

\section{Experiment 2 (No Attentional Set)}

1 Type of Item (Homogeneous Condition)

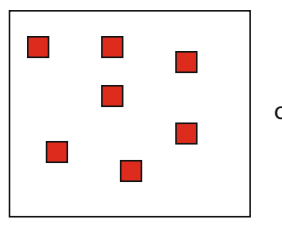

2 Types of Item

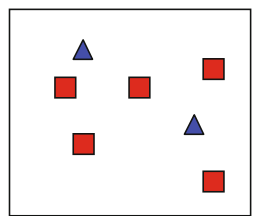

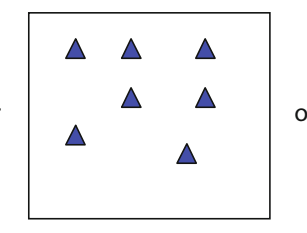

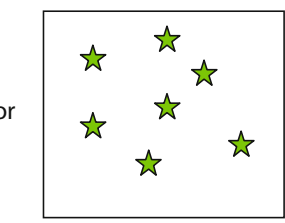

3 Types of Item

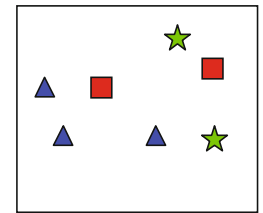

Figure 1. Sample displays (red squares, blue triangles, green stars) with 7 items in the homogeneous and heterogeneous conditions in Experiments $1 \mathrm{~A}$ and $1 \mathrm{~B}$, and in the 1- (homogeneous), 2-, and 3-types-of-item condition in Experiment 2. 
using different processes to enumerate the same number of items, for purposes of statistical analysis 1-3 items was considered the subitizing range and 6-8 items was considered the counting range, as has been done before (see also Trick \& Pylyshyn, 1993; Watson \& Maylor, 2006).

The RT data for Experiments 1A and 1B are presented in Figure 2. The results were typical of enumeration studies where subitizing and counting are evident. There were significant deviations from linearity in the enumeration function in every condition of every experiment $(p<.001)$, and, as shown in Table 1, significant differences in the RT slope between $1-3$ and $6-8$ items $(p<.05)$. Thus, the first prediction was supported: Manipulations of display heterogeneity do not prevent the emergence of subitizing and counting.

A necessary preliminary to determining the effects of heterogeneity is to ensure that there are no RT differences between homogeneous displays of the two types of item. When data from Experiments 1A and 1B were analyzed, there were no differences in RT for homogenous displays of red squares compared with blue triangles ( $M$ difference $=3 \mathrm{msec} ; S E$ of difference $=10 \mathrm{msec} ; F<1)$, and there were also no item $\times$ number interactions $(p>.1)$.

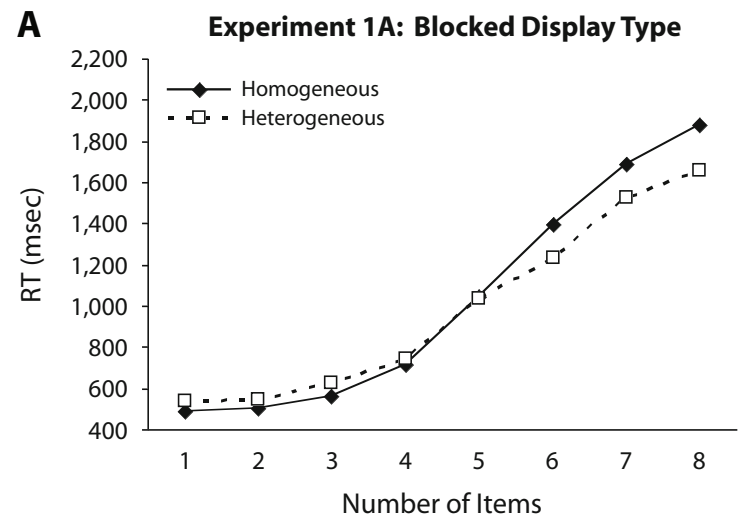

B Experiment 1B: Randomized Display Type

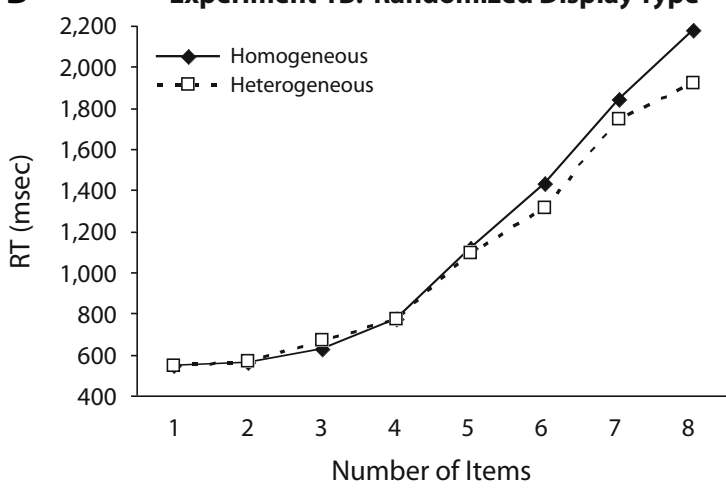

Figure 2. Mean enumeration latencies (in milliseconds) for homogeneous and heterogeneous displays in Experiments 1A and $1 \mathrm{~B}$. Figure $2 \mathrm{~A}$ presents the results of Experiment $1 \mathrm{~A}$, where the display manipulation was blocked. Figure $2 B$ presents the results of Experiment 1B, where the display manipulation was randomized.
Table 1

RT Slopes Expressed in Milliseconds/Item for All Experiments As a Function of Condition in the 1-3 and 6-8 Item Ranges

$$
\text { 1-3 Item Range 6-8 Item Range }
$$

Experiment 1A (attentional set, display manipulation blocked)

Homogeneous $\quad 36 \quad 249$

Heterogeneous $\quad 45 \quad 207$

Experiment 1B (attentional set, display manipulation randomized) $\begin{array}{lll}\text { Homogeneous } & 36 & 385 \\ \text { Heterogeneous } & 63 & 312\end{array}$ Heterogeneous $\quad 63 \quad 312$

Experiment 2 (no attentional set, display manipulation randomized) One type (homogeneous) $\quad 44 \quad 368$ $\begin{array}{lll}\text { Two types } & 60 & 330\end{array}$ Three types $41 \quad 306$

Experiment 3A (homogeneous displays only)

$\begin{array}{lll}\text { Flicker } & 42 & 297 \\ \text { Move } & 37 & 345 \\ \text { Switch color shape (CS) } & 34 & 291\end{array}$

$\begin{array}{lll}\text { Switch color shape (CS) } & 34 & 291 \\ \text { Move switch CS } & 30 & 315\end{array}$

Experiment 3B (attentional set, heterogeneous displays only) Flicker $\quad 20 \quad 183$

Move $\quad 24 \quad 154$

$\begin{array}{lll}\text { Switch CS } & 27 & 258\end{array}$

Move switch CS $16 \quad 292$

Experiment 3C (attentional set, heterogeneous displays only) Flicker $\quad 52 \quad 236$ Switch CS $\quad 52 \quad 279$

Switch S $\quad 55 \quad 282$

Switch C $\quad 53 \quad 245$

Novel CS $\quad 47 \quad 273$

The effect of item type was consistent across experiments, and there were no main effects or interactions involving experiment as a factor either $(F<1$ for all). Consequently, data for homogeneous displays of red squares and blue triangles were combined.

RT was then analyzed as a function of display (homogeneous, heterogeneous) and number (2-8). The results of Experiments $1 \mathrm{~A}$ and 1B were very similar, and to shorten the presentation, data from the two experiments were initially combined. Over the two studies, RT increased with the number of items $[F(1.37,30.1)=196.08, p<$ $\left..001, \eta^{2}=.90\right]$ and there was also an effect of display $\left[F(1,22)=7.89, p=.01, \eta^{2}=.26\right]$ and a crossover interaction [display $\times$ number, $F(2.69,59.19)=10.84, p<$ $\left..001, \eta^{2}=.31\right]$. As can be seen from Figure 2, the crossover occurred at 4 or 5 items, as might be expected if the transition between subitizing and counting occurred at 4 or 5. Experiment did not have an effect and did not interact with the other factors, which indicates that the blocking manipulation had little impact $(F<1$ for all).

Separate analyses were carried out in the subitizing and counting ranges in both experiments. In Experiment 1A, for $2-3$ items (the subitizing range), participants were $55 \mathrm{msec}$ slower to enumerate heterogeneous than to enumerate homogenous displays $\left[F(1,11)=10.21, p=.009, \eta^{2}=.48\right.$; $S E$ of the difference between homogeneous and heterogeneous conditions $=17 \mathrm{msec}$ ], and there was no display $\times$ number interaction within the $2-3$ item range $(p>.1)$. The detrimental effects of heterogeneity - or more properly, the expectation of heterogeneity that occurred because trials were blocked - were apparent even when there was only 1 item ( $M$ difference between the homogeneous and het- 
erogeneous condition $=49 \mathrm{msec} ; S E$ of the difference $=$ $26 \mathrm{msec}$ ). The minimal ( $6 \mathrm{msec}, p>.1)$ difference in the size of the heterogeneity effect for 1 item compared with that for 2 and 3 items suggests that an expectation for heterogeneity was enough to slow subitizing, even when there was no actual heterogeneity in the display. When 1-item displays were included, there was no display $\times$ number interaction in the $1-3$ range $(p>.1)$. In Experiment 1B, where trials were randomized, participants were $28 \mathrm{msec}$ slower to enumerate 2-3 heterogeneous items than they were to enumerate the same number of homogeneous items [display, $F(1,11)=5.35, p=.04, \eta^{2}=.33 ; S E$ of difference $=12 \mathrm{msec}]$. As before, there was no display $\times$ number interaction within the $2-3$ item range $(p>.1)$.

The predicted effect also emerged in the counting range (6-8 items). In Experiment 1A, participants were $182 \mathrm{msec}$ faster to enumerate 6-8 items when the items were heterogeneous [display, $F(1,11)=11.11, p<.007, \eta^{2}=.50$; LSD test of means, $p=.007 ; S E$ of difference $=55 \mathrm{msec}]$. Similarly, in Experiment 1B, participants were $158 \mathrm{msec}$ faster when items were heterogeneous [display, $F(1,11)=$ $8.87, p=.01, \eta^{2}=.45 ; S E$ of difference $\left.=53 \mathrm{msec}\right]$. There was no display $\times$ number interaction within the 6-8 item range in either experiment $(p>1)$. Overall, the difference between homogeneous and heterogeneous conditions was not quite as large in Experiment $1 \mathrm{~B}$ as it was in Experiment $1 \mathrm{~A}$, but this trend was not statistically significant.

\section{EXPERIMENT 2 \\ Enumerating Heterogeneous Items Without an Attentional Set to Select Items by Type}

In the first two experiments, it was argued that participants developed an attentional set to select items by type when enumerating heterogeneous items, and that this facilitated counting and impeded subitizing. It was proposed that this attentional set developed because participants were led to expect heterogeneous displays in which approximately half the items were red squares and the other half were blue triangles. However, in order to show that the result was produced by an attentional set to select items by type, rather than by heterogeneity per se, it was important to show that there are situations in which an attentional set to select items by type does not develop for heterogeneous displays. Therefore, in Experiment 2, the goal was to discourage the development of an attentional set to select items by type. One way to prevent this attentional set is to ensure that there would be no particular advantage to selecting items by type. If the relative number of red and blue items changed from trial to trial, addition would be no faster if participants selected by type than it would be if they selected by item location. However, an attentional set also reflects expectations, and it is possible that an attentional set might develop simply as the result of repeated trials where there were always the same two types of item together in heterogeneous displays. Thus, to further discourage the development of an attentional set, an additional type of item was included: green stars.

There are already studies that investigate the enumeration of heterogeneous displays with two types of item that differ in their features, where the relative number of each type could not be predicted from trial to trial. Both had participants enumerating red and green items, but they produced conflicting results. Puts and de Weert (1997) analyzed the time required to enumerate $3-5$ items when items were homogeneous (all red) rather than heterogeneous (a mixture of red and green items). They found that heterogeneity impeded enumeration of 3-5 items, and concluded that heterogeneity interferes with subitizing. In contrast, Watson and Maylor (2006) analyzed data in which participants were enumerating 2-3 items, a range across which subitizing definitely occurs in all adults, and in which the items were either homogeneous red, homogeneous green, or heterogeneous red and green. They found that it took significantly more time to enumerate green items than red, and that the difference between heterogeneous and homogeneous displays was actually the result of longer latencies to enumerate green items. They concluded that heterogeneity in item color had no effect on subitizing.

By using the three different types of item (red squares, blue triangles, and green stars), and presenting a range of numerosities that encompassed Watson and Maylor (2006) and Puts and de Weert (1997), it was possible to disentangle the effects of heterogeneity from those of numerosity and specific item types. This methodology also made it possible to evaluate another hypothesis in the enumeration literature: the idea that there would be some sort of advantage if the number of types equaled the number of tokens (e.g., Mozer, 1989).

Given Watson and Maylor's (2006) results, the prediction was that green items should take longer to enumerate than red, and that there should be no difference between latencies for homogeneous and heterogeneous displays of 2-3 items, once this color difference was taken into account. Second, based on Puts and de Weert (1997), it was predicted that with 4 or more items it should take longer to enumerate heterogeneous than homogeneous displays, even after the differences between red and green items were factored in. This is to be expected, if item heterogeneity disrupts grouping by proximity, and grouping starts to be important outside the subitizing range, where group-and-add counting begins. Finally, if type information facilitates token individuation, as suggested by Mozer (1989), participants should be faster and more accurate at enumerating 2 items when there are 2 different types of item than if there is only 1 type. Similarly, participants should be faster and more accurate at enumerating 3 items when there are 3 different types of item than they would be if there were only 2 types of item, or 1 type.

\section{Method}

Participants. Fourteen university students participated in the experiment (11 females). Participant characteristics were the same as in the other experiments, as was the method of payment.

Apparatus and Stimuli. Participants enumerated $1.27^{\circ} \times 1.27^{\circ}$ green stars $\left[\operatorname{RGB}(0,255,0), 16 \mathrm{~cd} / \mathrm{m}^{2}\right]$, as well as red squares and blue triangles. The relative number of red squares, blue triangles, and green stars varied from trial to trial, as shown in Figure 1. It was impossible to present every possible configuration of relative numbers of item and types of item without having considerably more trials in Experiment 2 than in Experiments 1A and 1B. In the interest of keeping the number of trials (and hence the amount of practice) rea- 
sonably similar between Experiment 2 and Experiments 1A and 1B, only a subset of all possible combinations of relative numbers and types was used. However, every type of item appeared equally often alone and in combination with every other type of item. See Table 2 for numbers of each type of item as a function of the total number of items and the total number of types of item in a given display.

Procedure. The display manipulation was randomized in Experiment 2 (as in Experiment 1B), and there were 1, 2, or 3 types of item in a given display. There were 324 experimental trials, but the procedure was otherwise identical to that of the earlier experiments

\section{Results and Discussion}

Data from one participant were dropped because of error rates in excess of $30 \%$ (there were initially 15 participants instead of 14). Overall, error rates were low $(M=2.6 \%)$ and though they increased with the number of items [number, $F(3.51,45.61)=4.01, p=.01, \eta^{2}=$ $.24 ; M$ error rates $=0.6 \%-5.5 \%]$, they did not vary with the type of item or number of different types of item in a display ( $p>.1$ for all). As in the previous experiments, RTs were dropped as outliers if they were beyond two $S D$ lengths of a participant's own mean for a given condition and number of trials $(4.6 \%$ of the trials were dropped on the basis of this criterion). Subitizing and counting were evident from RT slopes (Table 1).

The first analysis compared the RT required to enumerate red, blue, and green items. The results support Watson and Maylor (2006) in showing that it takes longer to enumerate homogeneous displays of green items than to enumerate homogenous displays of red items $(M$ difference $=38 \mathrm{msec}$; $S E$ of the differences $=18 \mathrm{msec}$; LSD, $p=.058$ ), a result that may reflect differences in brightness contrast between the two types of item and the background. The differences between red and blue items were minimal in this experiment, as they were in the first two experiments $(M$ difference $=$ $7 \mathrm{msec} ; S E$ of difference $=18 \mathrm{msec} ; \mathrm{LSD}, p>.1)$.

Following the procedure of Watson and Maylor (2006), latencies for the three separate types of homogeneous item were then compared with those for heterogeneous displays. These analyses revealed that when it came to enumerating two items, there were no significant differences among homogeneous displays of red, blue, and green items, and heterogeneous displays with two types of item $(F<1)$. When

Table 2

Relative Numbers of Items of Each Type in Experiment 2

\begin{tabular}{|c|c|c|}
\hline $\begin{array}{l}\text { No. of } \\
\text { Items }\end{array}$ & $\begin{array}{l}\text { No. of Types } \\
\text { of Item }\end{array}$ & Relative Nos. of Each Type of Item \\
\hline 2 & 1 or 2 & 2 types of item $(1 / 1)$ \\
\hline 3 & 1,2 , or 3 & $\begin{array}{l}2 \text { types of item }(2 / 1) \\
3 \text { types of item }(1 / 1 / 1)\end{array}$ \\
\hline 4 & 1,2 , or 3 & $\begin{array}{l}2 \text { types of item }(2 / 2 ; 3 / 1) \\
3 \text { types of item }(1 / 2 / 1)\end{array}$ \\
\hline 5 & 1,2 , or 3 & $\begin{array}{l}2 \text { types of item }(3 / 2 ; 4 / 1) \\
3 \text { types of item }(2 / 2 / 1)\end{array}$ \\
\hline 6 & 1,2 , or 3 & $\begin{array}{l}2 \text { types of item }(3 / 3 ; 4 / 2 ; 5 / 1) \\
3 \text { types of item }(2 / 2 / 2 ; 3 / 2 / 1)\end{array}$ \\
\hline 7 & 1,2 or 3 & $\begin{array}{l}2 \text { types of item }(4 / 3 ; 5 / 2 ; 6 / 1) \\
3 \text { types of item }(3 / 2 / 2 ; 4 / 2 / 1)\end{array}$ \\
\hline 8 & 1,2 or 3 & $\begin{array}{l}2 \text { types of item }(4 / 4 ; 5 / 3 ; 6 / 2) \\
3 \text { types of item }(3 / 3 / 2 ; 4 / 3 / 1)\end{array}$ \\
\hline
\end{tabular}

the latencies for red, blue, and green items were averaged, the difference between homogeneous and heterogeneous displays was $19 \mathrm{msec}$ ( $S E$ of difference $=7 \mathrm{msec}$ ), a difference half the size of the average difference between homogeneous displays of red and green items. Similarly, when there were 3 items, there were no significant differences among the five conditions (the mean times to enumerate homogeneous displays of red squares, blue triangles, and green stars, and heterogeneous displays made up of 2 and 3 types of item, $F<1$ ). When latencies for red, blue, and green items were averaged, the difference between 1 and 2 types of item was only $22 \mathrm{msec}$ ( $S E$ of difference $=13 \mathrm{msec}$ ), once again less than the average difference between red and green items. Thus, the results of the present experiment replicate Watson and Maylor (2006) in showing that display heterogeneity has no effect in the 2-3 item range. There was no support for Mozer's (1989) contention that enumeration would be faster if the number of types of item equaled the number of items though, as shown in Figure 3.

The present results support Watson and Maylor (2006) for 2 and 3 items, a range in which subitizing definitely takes place in adults, but they support Puts and de Weert (1997) outside that range, particularly in the transition zone (4-5 items, where some might be expected to subitize and others to count). Once there were more than 3 items, the magnitude of the heterogeneity effects was larger than were the differences between red and green items, and significant effects emerged when homogeneous displays of the three types were compared with heterogeneous displays $(p<.05)$. Consequently, to simplify presentation, latencies for homogeneous displays of red squares, blue triangles, and green stars were combined to form a unified average for homogeneous displays. When there were 4 and 5 items, heterogeneity interfered significantly with enumeration [display, $F(2,26)=4.95, p=.02, \eta^{2}=.28$ ], with the maximal difference between displays with $1 \mathrm{com}-$ pared with 3 types of item $[M$ difference $=82 \mathrm{msec} ; S E$ of difference $=27 \mathrm{msec} ; \mathrm{LSD}, p=.01]$. Heterogeneity also interfered in the 6-8 item range, though the effect was only marginal [display, $F(2,26)=2.20, p=.13, \eta^{2}=.15$ ], and the maximal difference was between 1 and 2 types of item $(M$ difference $=74 \mathrm{msec} ; S E$ of difference $=42 \mathrm{msec}$; LSD test of means, $p=.099$ ). Furthermore, as might be expected from Puts and de Weert, in the 4-8 item range, where heterogeneity had a negative effect, there was no significant difference in the time required to enumerate 2 as opposed to 3 types of item ( $F<1 ; M$ difference between displays with 2 and 3 types of items $=18 \mathrm{msec} ; S E$ of difference $=25 \mathrm{msec}$ ). This is because any heterogeneity in item features disrupts grouping by proximity, so the damage is done by the time there are 2 types of item.

Although in this study heterogeneity seems to slow the enumeration of larger numbers of items rather than speed it (as it did in the first two experiments), the effect seems weak and erratic. This may be because of the ways in which the similar items were distributed in specific displays. If similar items are grouped, such groupings could either support or conflict with the organization provided by grouping by proximity depending on whether or not similar items were close together. Consequently, hetero- 
geneity could either speed or slow enumeration depending on the specific way similar items were clustered (as shown in Beckwith \& Restle, 1966), though, on average, random configurations should interfere with grouping by proximity because the probability of all the similar items appearing close together would be low.

Overall, the results of Experiment 2 are clear: They support Watson and Maylor (2006) and Puts and de Weert (1997). Heterogeneity did not impede enumeration of $2-3$ items, or speed the enumeration of larger numbers of items. This pattern of results is very different from the one observed in Experiments 1A and 1B (see Table 3 for a summary).

However, the most dramatic way to demonstrate the impact of expectations involves comparing results from the two experiments where the display manipulation was randomized: Experiment 1B (where participants expected approximately equal numbers of red squares and blue triangles in every heterogeneous display) and Experiment 2 (where they did not). First, a comparison was made between RTs to enumerate homogeneous displays of 1-8 items, where the items were red squares or blue triangles. There was no significant difference between latencies for Experiment 1B and Experiment 2 (Experiment $1 \mathrm{~B}$ latencies were greater by $15 \mathrm{msec})$ nor were there any interactions $(F<1$ for all). It seems to make little difference whether participants expect two types of item or three when they enumerate homogeneous displays of red squares or blue triangles.

The analyses of the effects of expectations on the enumeration of heterogeneous displays of red squares and blue triangles, where approximately half the items were of one type and half of the other, was more difficult to carry out. In Experiment 2, to prevent an attentional set it was necessary to have a variety of different types of item in combination, and different relative numerosities of each type, so that no particular combination of item types or relative numerosities occurred more often than any other in heterogeneous displays. Nonetheless, to get reliable RT data it is necessary to have a reasonable number of trials with each item combination and relative number, and the only way to do that and still prevent an attentional set would be to expose participants in Experiment 2 to a very large number of trials, making them more practiced and thus creating a confound.

As a result, the best that could be done was an exploratory analysis, where data from the few trials where there were approximately equal numbers of red squares and blue triangles in Experiment 2 were selected out. Trials of this type were found for 2 and 3 items and for 7 and 8 items (although for some comparisons there were as few as $2-4$ RTs per participant per number). When there were $2-3$ red squares and blue triangles, participants were $57 \mathrm{msec}$ slower to enumerate heterogeneous displays when there was an attentional set than when there was not. (Enumeration of 2-3 heterogeneous items was faster in Experiment 2 than in Experiment 1B.) In contrast, when there were 7-8 red squares and blue triangles, participants were $166 \mathrm{msec}$ faster to enumerate heterogeneous displays when there was an attentional set. (Enumeration of 7-8 heterogeneous items was slower in Experiment 2 than in Experiment 1B.) These are only trends $(p>.05)$. There weren't enough RTs to make statistically reliable comparisons (variability is high, with only 2-4 RTs
Experiment 2: Varying Numbers of Each Type

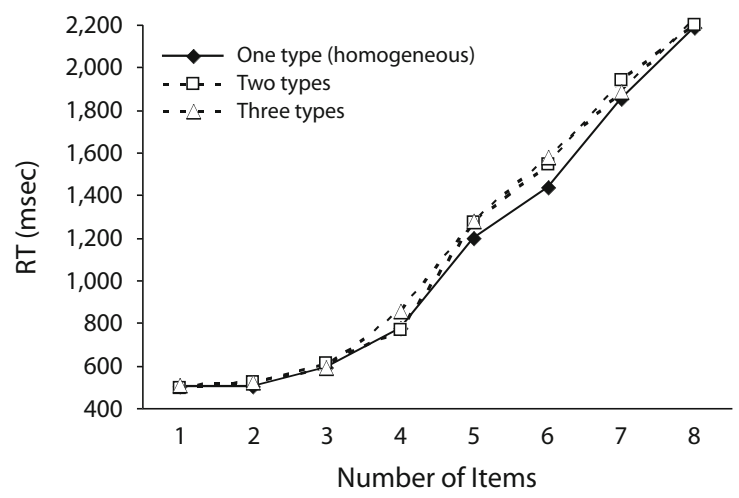

Figure 3. Mean enumeration latencies (in milliseconds) for Experiment 2 . In this experiment there was one type of item (homogeneous condition), two types of item, or three types of item with varying numbers of each type.

per mean per individual), but these results suggest that heterogeneous displays with approximately equal numbers of red squares and blue triangles are enumerated differently, depending on participant expectations.

\section{EXPERIMENT 3 Feature Change and Enumeration}

The previous experiments showed that heterogeneity in item features can have different effects, depending on whether or not participants formed an attentional set to select items by type. However, the next question that arises is whether an attentional set to select by type is resistant to moment-to-moment change in items, an issue of relevance to understanding how selection and individuation are accomplished. In particular, if items are continually trading features from moment to moment, would selection by type no longer be possible? If so, then data should follow the pattern shown in Experiment 2: Heterogeneity should impede enumeration slightly in the counting range (6-8 items) and have no effect in the subitizing range (2-3 items). Alternatively, if participants continue to select items by type in the

Table 3

Comparison of the Effects of Display Type (in Milliseconds)

\begin{tabular}{|c|c|c|}
\hline Exper & 2-3 Items & \\
\hline \multicolumn{3}{|c|}{$\begin{array}{l}\text { Experiment 1A (display type manipulation blocked; attentional set) } \\
\qquad-55 \mathrm{msec}^{*} \\
+182 \mathrm{msec}^{*}\end{array}$} \\
\hline \multicolumn{3}{|c|}{$\begin{array}{l}\text { Experiment 1B (display type manipulation randomized; attentional set) } \\
\qquad-28 \mathrm{msec}^{*} \\
+158 \mathrm{msec}^{*}\end{array}$} \\
\hline \multicolumn{3}{|c|}{$\begin{array}{r}\text { Experiment } 2 \text { (display type manipulation randomized; no attentional set) } \\
--^{\dagger}-74 \operatorname{msec}(p=.099)\end{array}$} \\
\hline \multicolumn{3}{|c|}{$\begin{array}{l}\text { periment } 3 \mathrm{~A} \text { (cross-experiment comparison; homogeneous items) } \\
\text { and Experiment } 3 \mathrm{C} \text { (heterogeneous items; attentional set) }\end{array}$} \\
\hline & & \\
\hline & & \\
\hline
\end{tabular}

Note - "+" indicates that heterogeneity speeds enumeration; "-" indicates that heterogeneity slows enumeration. "Difference less than the difference between homogeneous displays of red and green items. ${ }^{*} p<.05$. 
face of transients and feature change, heterogeneity should speed enumeration in the counting range and impede it in the subitizing range, as shown in Experiments 1A and 1B. Three interrelated experiments were performed, first to determine how various types of transient and feature change affected enumeration in general, then to ascertain whether these factors influenced whether participants select items by type when enumerating heterogeneous displays.

The impact of different types of item change were examined as they affected the enumeration of displays where items were homogeneous (Experiment 3A) or heterogeneous (Experiments 3B and 3C). Participants were required to enumerate items that were continually changing, repeatedly cycling through a series of stages until a response was made. Items always started out as red squares or blue triangles but then changed in various ways; nonetheless, they passed through all their possible forms before even the fastest participant could enumerate 1 item. In some conditions one or more features changed, and in others the items maintained their properties and simply flickered or moved within a small area (see Figure 4). The latter two conditions were used to distinguish the effects of feature change from those of image transients.

A primary prediction was that the deviations in RT slope indicative of subitizing and counting would occur in every condition of every experiment, as might be expected if heterogeneity and momentary transients did not prevent subitizing. The processes used to define and individuate items for purposes of enumeration do not require that items maintain their features (Trick \& Pylyshyn, 1994).

Although the main prediction was simple, the others were more complex, so these will be developed in separate sections for purposes of clarity. The first two are on the impact of item change and the last is on how item change influences the effects of display heterogeneity and attentional set.

\section{Enumerating Homogeneous Items That Change (Experiment 3A)}

Participants enumerated homogeneous displays where items could flicker or wiggle within a restricted area (flicker and move conditions), trade colors and shapes (switch CS), or move and trade colors and shape (move switch CS). The prediction was that change type would have no effect when displays were homogeneous. Participants should take no longer to enumerate items that changed in two dimensions (switch CS and move switch CS conditions) than to enumerate items that retained their features and changed in other ways (flicker and move conditions). Change type was not expected to have an effect, because the features of selected items should only influence latencies when they are relevant to selection and when all of the items in a display are the same; there is no to way to select items based on their features. Therefore, RTs should increase with the number of items, but there should be no change type $\times$ number interaction.

\section{Enumerating Heterogeneous Items That Change (Experiments 3B and 3C)}

In Experiments 3B and 3C, participants were presented with displays featuring two types of item. Approximately half the items were of one type and half the other, a situation that encouraged selection by type in Experiments 1A and 1B. In Experiment 3B, participants enumerated items that flickered, moved, switched color and shape, or moved and switched color and shape, as in Experiment 3A. In Experiment $3 \mathrm{C}$, the two movement-control conditions were dropped to make way for three new conditions: switch color, switch shape, and novel color and shape (switch C, switch S, novel CS). See Figure 4.

Hypotheses varied depending on the number of items. It was predicted that when there were 6-8 heterogeneous items (too many to individuate all at once, given the limited number of FINSTs), it should be possible to select items by type when they retain their features, and thus profit from the reduction in uncertainty that occurs when there are always approximately equal numbers of each type. Consequently, it should take less time to enumerate 6-8 items when items retain their features (flicker and move conditions) than when they trade features (switch CS and move switch CS conditions). Feature change should have an impact in the counting range with heterogeneous items, and in particular, the more features that change the more interference there should be. It should be harder to select items by type if the items changed in terms of both their color and shape, than it should be if the items only changed in color or only changed in shape (switch $\mathrm{C}$ and switch $\mathrm{S}$ conditions). That is because participants could still select items based on one dimension even if there were changes in the other. However, it should be no more difficult to enumerate items that traded colors and shapes (switch CS) than items that take on new colors and shapes (novel CS). When items change on both dimensions there should be no inducement to select items by type, and therefore it should not matter which features the items take on.

In contrast, change type should have no effect in the subitizing range (1-3 items). That is because when there are fewer than 4 items, items can be individuated all at once within the first frame of the display (even though producing a response takes longer). FINSTs, the mental reference tokens that permit rapid enumeration in the subitizing range, do not represent the properties of the items to which they refer, and they stay assigned despite changes in item properties and position, so there was no reason to expect change type would affect enumeration in the subitizing range (Pylyshyn, 1989, in press).

\section{Comparing the Effects of Item Heterogeneity When Items Change in Different Ways}

In Experiments $1 \mathrm{~A}$ and $1 \mathrm{~B}$, when participants saw displays with two types of item (approximately half of one type and half of the other), they were slower to enumerate 1-3 items but faster to enumerate 6-8 items, compared with when they were enumerating homogeneous items. The interpretation was that when participants repeatedly saw heterogeneous displays such as these, they adopted a strategy of selecting items by type when enumerating (i.e., first enumerate one type, then the other). If participants continued to adopt this strategy when items change features, the same pattern of results should occur for flickering items and items that trade color and shape. Therefore, 


\section{A}

Experiment 3A: Homogeneous Items (Half of the Trials Have Red Squares to Start)

Switch CS (Switch Color and Shape)

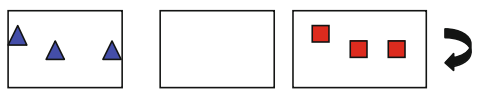

Flicker
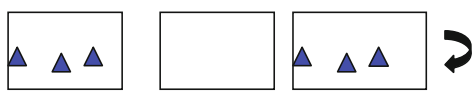

Move switch CS is the same as switch CS, except each also moves within $0.8^{\circ}$ area

Move is the same as flicker, except each item also moves within a $0.8^{\circ}$ area

\section{B}

Experiment 3B: Heterogeneous Items (More Red Items in Half of the Trials)

Switch CS (Switch Color and Shape)
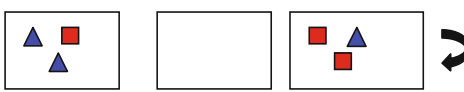

Flicker
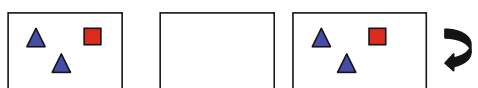

Move is the same as flicker, except each item also moves within a $0.8^{\circ}$ area

\section{C}

Experiment 3C: Heterogeneous Items (More Red Items for Half of the Trials)

Switch C (Switch Color)

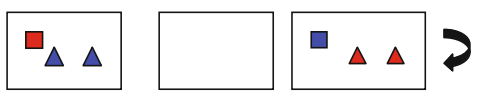

Switch S (Switch Shape)

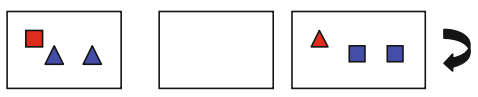

Novel CS (Novel Color and Shape)
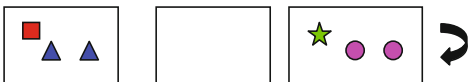

Figure 4. Sample displays with 3 items for the conditions in Experiments $\mathbf{3 A}-\mathbf{3 C}$. Items always started out as blue triangles or red squares, though these items could change into blue squares, red triangles, green stars, or mauve circles, depending on the condition. Each display involved a series of individual frames in which the items came on for $101 \mathrm{msec}$ (frame one in the diagram), disappeared for $61 \mathrm{msec}$ (frame two, the blank square in the diagram), and then reappeared for another $101 \mathrm{msec}$ (frame three). This sequence of frames then repeated, and continued repeating until a response was made. In some conditions the items changed features from the first to third frame. In others they stayed the same or merely moved.

there should be a display $\times$ number interaction when the results of Experiments 3A (homogeneous items) and 3C (heterogeneous items) are analyzed together, but no display $\times$ number $\times$ change interaction.

In contrast, if participants abandon selection by type in heterogeneous displays when items change features there should be a display $\times$ number $\times$ change interaction. The crossover interaction where heterogeneity impedes subitizing and facilitates counting should be seen for flickering items (flicker condition) but not for items that trade colors and shapes (switch CS).

\section{Method}

Participants. There were 69 observers (52 females): 30 in Experiment 3A, 9 in Experiment 3B (a pilot study), and 30 in Experiment $3 \mathrm{C}$, and none had been in any of the previous experiments. None of the participants had been diagnosed with epilepsy. Overall participant characteristics were the same as in the earlier experiments.

Stimuli. Participants enumerated colored figures that changed from moment to moment (101 msec in one form; $61 \mathrm{msec}$ blank; $101 \mathrm{msec}$ in the other) until the spacebar was pressed to terminate the display. In switch CS, items switched both color and shape, which is to say that red squares turned into blue triangles and blue triangles turned into red squares. In the flicker condition, items flickered on and off (101 msec on, $61 \mathrm{msec}$ off) but retained their features. In 
the move condition, each item shifted randomly within an area that was $0.80^{\circ}$ from the starting point through four frames of movement. Items repeatedly came on for $101 \mathrm{msec}$, disappeared for $61 \mathrm{msec}$, then came on in a different position for $101 \mathrm{msec}$. They resumed their starting position after four frames and the cycle began again. Finally, in the move switch CS condition, items moved and switched both their color and shape until a response was made.

The only factor that differentiated Experiments $3 \mathrm{~A}$ and $3 \mathrm{~B}$ was display heterogeneity. In Experiment $3 \mathrm{~A}$, all the items within a given display were homogeneous (all red squares or all blue triangles). In half of the trials, items started out as red squares, and in the other half they started out as blue triangles. In Experiment 3B, the displays were heterogeneous; approximately half the items were red squares and half were blue triangles.

In Experiment 3C, participants once again enumerated heterogeneous displays, but the two movement controls were dropped to make way for three new conditions: switch $\mathrm{S}$ (items switched shape but not color), switch $\mathrm{C}$ (items switched color but not shape), and novel CS (items took on colors and shapes that had not been in the initial display). Within each block of trials, there was never uncertainty about how items would change. For example, in the switch S (shape) condition, red squares always turned into red triangles and blue triangles turned into blue squares. In the switch $\mathrm{C}$ (color) condition, red squares became blue squares and blue triangles became red triangles. In the novel CS condition, red squares always turned into green stars and blue triangles always turned into mauve circles. Display configurations in Experiments $3 \mathrm{~A}-3 \mathrm{C}$ were the same as in the first three experiments, but there was one new stimulus: a $1.02^{\circ}$ diameter mauve circle [RGB $\left.(255,0,255), 23 \mathrm{~cd} / \mathrm{m}^{2}\right]$.

Procedure. For all studies, change type was blocked and order counterbalanced in such a way that each condition occurred first an approximately equal number of times. Before each change condition block, participants were given 18 trials of practice for the relevant condition. In each experiment, each participant viewed 108 trials of each type of change, which meant there were a total of 432 trials in Experiments 3A and 3B, and 540 trials in Experiment 3C. Participants were told that the items might change and move, but otherwise the procedure was the same as in the earlier studies.

\section{Results}

In Experiments 3A, 3B, and 3C, enumeration latencies and error rates were analyzed as a function of number and change type. As before, data from participants with error rates in excess of $30 \%$ for any number and condition were dropped from the analysis, and the participants were replaced. This resulted in the loss of data from 2 participants in Experiment 3A and 1 in Experiment 3C. For each individual, for each condition and number of items, latencies further than $2 S D$ s from that individual's respective mean for that condition and number were dropped. This resulted in the loss of 4.4\%, 4.2\%, and 4.4\% of the trials in Experiments $3 \mathrm{~A}-3 \mathrm{C}$, respectively.

The initial prediction was supported. Table 1 shows that in every condition of all three experiments, there were differences in the RT slope between the 1-3 and 6-8 item range $(p<.05)$, and significant deviations from linearity when RT was analyzed as a function of number $(p<.001$ for all). For purposes of clarity, the remainder of the results will be reported in separate sections, one for displays that were homogeneous (Experiment 3A), one for displays that were heterogeneous (Experiments $3 \mathrm{~B}$ and $3 \mathrm{C}$ ), and one for the cross-experiment comparison that was necessary to determine whether display heterogeneity had the same effects regardless of how items changed.

\section{Experiment 3A: Enumerating Homogeneous Items That Change}

The effects of four types of change (flicker, switch CS, move, and move switch CS conditions) were measured as a function of number (1-8 items). Error rates were low $(M=2.5 \%)$, although they increased with the number of items $\left[F(2.63,76.28)=9.73, p<.001, \eta^{2}=.25, M\right.$ errorrate range $=1.0 \%-5.6 \%]$. As predicted, the effects of item change were minimal when the items were homogeneous. Change type had no effect on error rates, and there was no change $\times$ number interaction $(F<1$ for both).

Number had its usual effect, with RT increasing with the number of items $[F(1.41,40.93)=349.62, p<.001$, $\left.\eta^{2}=.92\right]$, as shown in Figure 5A. As predicted, given that item features are not relevant when all the items in the display have the same features, participants were no slower to enumerate items that were trading features (switch CS, move switch CS) than items that retained their features and simply flickered or moved $(F<1)$. Overall, change type had no effect latencies $(F<1)$, and there was also no change $\times$ number interaction $(p=.24)$.

\section{Experiments 3B and 3C: Enumerating Heterogeneous Items That Change}

Experiment 3B. There were four types of item change in this experiment, as in Experiment 3A (flicker, switch CS, move, and move switch CS). The error rates were higher than in our previous experiments (4.8\%). Exaggerated end effects were apparent; error rates were between two and four times higher than those for 8 items in other studies (e.g., $M$ error rate $=14.8 \%, 21.3 \%, 7.41 \%$, and $18.5 \%$, in the flicker, switch CS, move, and move switch CS conditions, respectively). As always, errors increased with the number of items $[F(1.99,15.92)=13.36, p<.001$, $\eta^{2}=.63, M$ error-rate range $\left.=0.2 \%-15.5 \%\right]$, but there was also a change $\times$ number interaction $[F(21,168)=$ $\left.3.71, p<.001, \eta^{2}=.32\right]$ that did not replicate in Experiment $3 \mathrm{C}$, which also had heterogeneous displays. As can be seen from Figure 5B and Table 1, RT and RT slopes were suspiciously low in this experiment; it seems probable that the participants were trading speed for accuracy, especially in the counting range.

Nonetheless, the results will be reported for two reasons: First, they support the general trends observed in Experiment $3 \mathrm{C}$; second, they provide a justification for dropping the two movement-control conditions (move and move switch CS). In Experiment 3B, change had a significant effect on RT $\left[F(3,24)=5.92, p=.004, \eta^{2}=.43\right]$, and there was a significant change $\times$ number interaction $\left[F(21,168)=4.82, p=.008, \eta^{2}=.38\right]$. As predicted, there were no significant main effects or interactions involving change in the subitizing range $(p>.1)$. In the counting range (6-8 items), change had the expected effect on enumeration latencies [change, $F(3,24)=7.16, p=$ $\left..001, \eta^{2}=.47\right]$. The switch CS condition took $263 \mathrm{msec}$ longer than the flicker condition did ( $S E$ of difference $=$ 119; LSD, $p=.058$ ). Similarly, switch CS move condition took 310 msec longer than the move condition (SE of difference $=65$ msec; LSD, $p=.001)$. The effects of item 
A

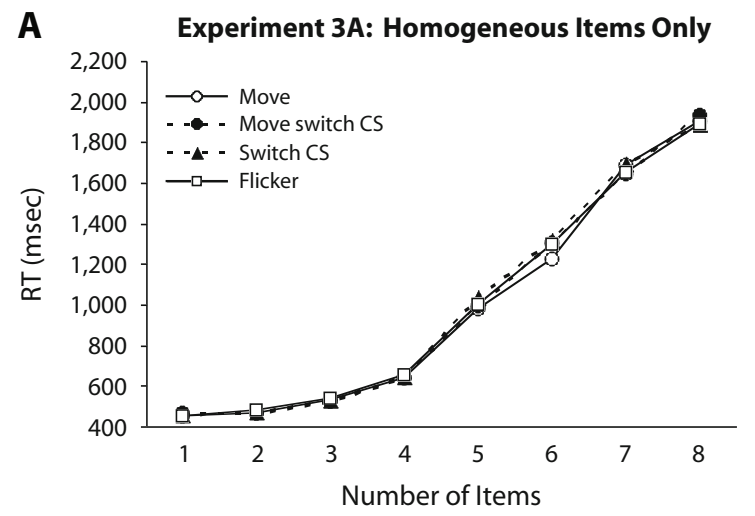

B

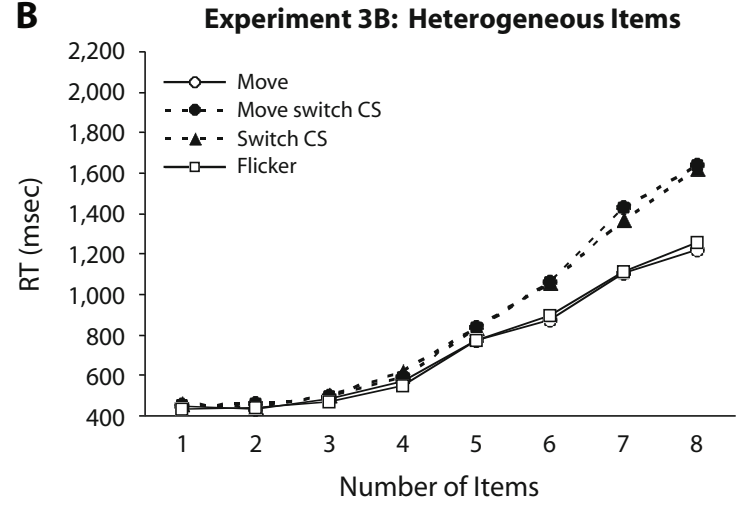

C Experiment 3C: Heterogeneous Items

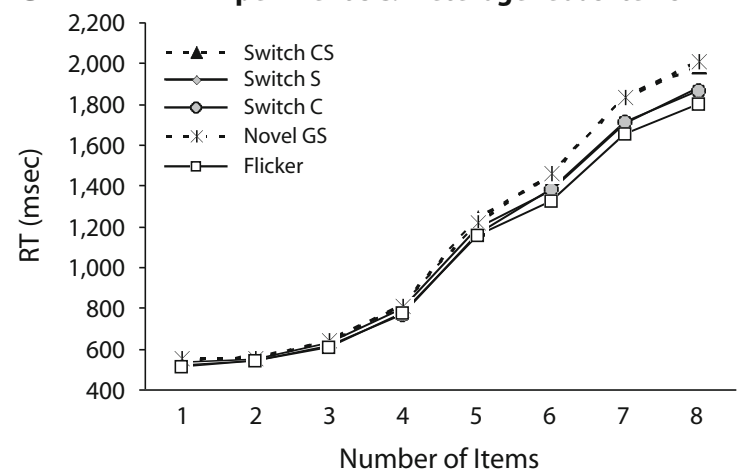

Figure 5. Mean enumeration latencies (in milliseconds) as a function of the type of moment-to-moment item change in Experiments $3 \mathrm{~A}-3 \mathrm{C}$ are listed in Figures $5 \mathrm{~A}-5 \mathrm{C}$, respectively. In Figure 5A, the task was to enumerate homogeneous items that always started out as red squares or blue triangles. In Figures 5B and 5C, the task was to enumerate displays of heterogeneous items, when all items started out as red squares or blue triangles, but in any given display approximately half of the items were red squares and the other half blue triangles. In some conditions, items would change color, shape, or both.

movement were minimal. RT for flicker and move conditions differed by only $21 \mathrm{msec}$ in the 6-8 item range (SE of difference $=51 \mathrm{msec} ; p>.1)$. Similarly, RT for switch CS and move switch CS differed by only $27 \mathrm{msec}$ (SE of difference $=103 \mathrm{msec}, p>.1)$. There was a marginal change $\times$ number interaction within the 6-8 item range $\left[F(3.17,25.37)=2.54, p=.08, \eta^{2}=.24\right]$, although this result was not replicated in later experiments; it may be the result of a speed-accuracy trade-off at the top of the range, where the error rates were especially high.

Experiment 3C. There were five change conditions: flicker; switch color and shape; switch color; switch shape; and novel color and shape (see Figure 4). Nonetheless, error rates were in line with those of earlier studies ( $M$ error rate $=3.6 \%$ ), although as always, errors increased with the number of items $\left[F(2.10,61.11)=14.79, p<.001, \eta^{2}=\right.$ $.34 ; M$ error-rate range $=0.9 \%-8.4 \%$ ]. In this experiment, change had no effect on error rates, and this factor did not enter into any interactions $(F<1$ for all).

RTs are presented in Figure 5C. Latencies increased with the number of items [number, $F(1.71,49.79)=388.751$, $\left.p<.001, \eta^{2}=.93\right]$, and the effects of the type of item change were significant [change, $F(2.36,68.41)=3.82$, $\left.p=.021, \eta^{2}=.12\right]$. As in Experiment $3 \mathrm{~B}$, there was also a change $X$ number interaction, but in this case it was only marginal, with Greenhouse-Geisser corrected degrees of freedom $\left[F(7.38,214.0)=1.84, p=.078, \eta^{2}=.06\right]$.

As predicted, when the 1-3 item range was examined, change type had no significant effect on latencies. Nor were there any change $\times$ number interactions within this range $(p>.1)$, although change type did have significant effects when there were $6-8$ items $[F(2.67,77.47)=$ $\left.4.04, p=.013, \eta^{2}=.12\right]$. As can be seen from Figure 5C, RTs were highest in the switch CS condition (where red squares switched with blue triangles) and in the novel CS condition (where red squares turned into green stars and blue triangles turned into mauve circles). As predicted, latencies for the switch CS and novel CS were similar; in fact, they were within $4 \mathrm{msec}$ of each other ( $S E$ of difference $=50 \mathrm{msec} ; p=.93)$. There was also little difference between the switch shape and switch color conditions in the 6-8 item range ( $M$ difference $=2 \mathrm{msec}$; $S E$ of difference $=35$ msec; $p=.95$ ).

To simplify calculations, in the 6-8 item range, switch $\mathrm{CS}$ and novel CS conditions were combined to form a twodimension-change condition and the switch $\mathrm{S}$ and switch $\mathrm{C}$ conditions were combined to form a one-dimensionchange condition. These averages were compared with the flicker condition $\left[F(2,58)=6.84, p=.002, \eta^{2}=.19\right]$. LSD analysis revealed that the two-dimension-change latencies were significantly greater than those for the flicker condition $(M$ difference $=177 \mathrm{msec} ; S E$ of difference $=$ $45 \mathrm{msec}, p=.001)$, and marginally greater than those for the one-dimension-change condition ( $M$ difference $=$ $109 \mathrm{msec}$; $S E$ of difference $=56 \mathrm{msec}, p=.061)$. Onedimension-change latencies were $68 \mathrm{msec}$ longer than those in the flicker condition, but this difference was not large enough to achieve statistical significance in the 6-8 item range ( $S E$ of difference $=42 \mathrm{msec}, p=.12$ ).

\section{Comparing the Effects of Item Heterogeneity When Items Change in Different Ways}

In the earlier experiments, when items were heterogeneous and approximately half were of one type and half of 
another, enumeration in the subitizing range was slowed and enumeration in the counting range was expedited. In order to find out whether this occurred when items flickered and changed features, the results from Experiment $3 \mathrm{~A}$ (homogeneous items) and Experiment 3C (heterogeneous items) were compared. RT data were analyzed as a function of display (homogeneous, heterogeneous), change (flicker, switch CS), and number (1-8), with display as a betweensubjects factor. (Error analyses are not reported, because number was the only factor that influenced error rates.)

The RT data show a three-way interaction [display $\times$ change $\times$ number, $F(7,406)=2.27, p=.028, \eta^{2}=$ $.04]$, which suggests that display heterogeneity had different effects, depending on how many items there were and how the items changed. To explore the interaction, RT data for the flicker and switch color shape trials were analyzed separately. As predicted, in the flicker condition, where items retained their features, the overall pattern of results was similar to that in Experiments $1 \mathrm{~A}$ and $1 \mathrm{~B}$ in that there was a crossover interaction [display $\times$ number, $\left.F(7,406)=2.80, p=.007, \eta^{2}=.05\right]$. This suggests that participants were adopting a strategy of selecting items by type. Heterogeneity slowed enumeration in the subitizing range and sped it in the counting range (the crossover occurred after 5 items). Specifically, when there were 2-3 flickering items, it took $63 \mathrm{msec}$ longer to enumerate heterogeneous items as opposed to homogeneous ones $\left[F(1,58)=4.45, p=.039, \eta^{2}=.07 ; S E\right.$ of difference $=$ $30 \mathrm{msec}]$. This trend was even apparent when there was only a single item ( $M$ difference $=47 ; S E=28)$, and the size of this difference was comparable to that observed with static items in Experiment $1 \mathrm{~A}$ (where $M$ difference = 49). The difference between the homogeneous and heterogeneous conditions was not quite as large for 1 item as it was for 2 and 3 , but when 1-item trials were included with the 2- and 3-item trials, there was no number $\times$ display type interaction $(F<1)$.

In the 6-8 item range, participants enumerated heterogeneous items faster than they did homogeneous ones, though in this case the difference was only $41 \mathrm{msec}$ ( $S E$ of difference $=95 \mathrm{msec} ; p>.1$ ), which suggests that it may be more difficult to benefit from selection by type when there are image transients. Although the display $X$ number interaction did not achieve statistical significance inside the 6-8 item range $[F(2,116)=2.16, p=.13]$, in this experiment, the large $(\geq 100 \mathrm{msec})$ benefits of display heterogeneity did not emerge for flickering items until there were 8 items in the display.

Results were different in the switch CS condition than in the flicker condition, but the pattern was not quite the same as that observed in Experiment 2, where there was no inducement to select items by type when enumerating heterogeneous items (and heterogeneity impeded enumeration slightly in the counting range, and had no effect in the subitizing range). Heterogeneity impeded the enumeration of 6-8 items in the switch CS condition; surprisingly, it also impeded the enumeration of $2-3$ items. There was a main effect of display $[F(1,58)=4.37, p=.041$, $\left.\eta^{2}=.07\right]$ but no display $\times$ number interaction $(F<1)$. Heterogeneous items required $117 \mathrm{msec}$ longer to enu- merate than did homogeneous items ( $S E$ of the difference $=56 \mathrm{msec}$ ). Interestingly, the difference between groups expecting homogeneous and heterogeneous items was also apparent when there was only a single item in the display [display, $F(1,68)=6.17, p=.016, \eta^{2}=.10 ; M$ difference $=69 \mathrm{msec} ; S E$ of the difference $=28 \mathrm{msec}]$.

A final analysis was carried out to determine whether the effects of heterogeneity were of the same magnitude in the flicker and switch CS conditions for the subitizing and counting ranges. Given that within both the subitizing range ( $2-3$ items) and the counting range (6-8 items) there were no significant interactions between condition and number, latencies were averaged within each range to simplify calculations. Averaged RTs were then analyzed as a function of display (homogeneous, heterogeneous) and change (flicker, switch CS). Results are presented in Figures 6A and 6B for the subitizing and counting ranges, respectively. In the subitizing range, homogeneous items were enumerated faster than heterogeneous ones were, regardless of the way in which items changed $\left[F(1,58)=7.29, p=.009, \eta^{2}=.11\right]$, and the display $X$ change interaction was not statistically significant $[F(1,58)=2.35, p=.13]$.

In contrast, there was a display $\times$ change interaction when average latencies to enumerate 6-8 items were analyzed $\left[F(1,58)=7.50, p=.008, \eta^{2}=.11\right]$, as shown in Figure 6B. In this range, heterogeneity slowed enumeration in the switch CS condition (by $111 \mathrm{msec}$, on average) and sped it in the flicker condition (by $41 \mathrm{msec}$, on average).

\section{GENERAL DISCUSSION}

These experiments show that heterogeneity and item change can have dramatically different effects on subitizing and counting - or at least enumeration of small and large numbers of items in situations where subitizing and counting are evident from differences in RT slope in the 1-3 and 6-8 item range. Moreover, these experiments show that heterogeneity can have markedly different effects, depending on expectations and item change. Results will first be discussed as they relate to selection, then more broadly as they relate to enumeration.

\section{Subitizing, Counting, and Attentional Selection}

Overall, these results are a tribute to the flexibility of the process that defines and individuates items for purposes of subitizing and counting. Items could be homogeneous or heterogeneous, items could change or move, and the discontinuity in RT slope that is the trademark of the transition between subitizing and counting was always evident, as shown in Table 1. If participants were using item features to distinguish between items, homogeneous items would be difficult, and items that constantly changed color and shape would be especially problematic. However, subitizing and counting emerged despite homogeneity and change, and participants could enumerate homogeneous items that changed as efficiently as they enumerated items that retained their features. This result is to be expected if the process that individuates items does not use item properties to distinguish between them (Pylyshyn, in press; Trick \& Pylyshyn, 1994). 
A

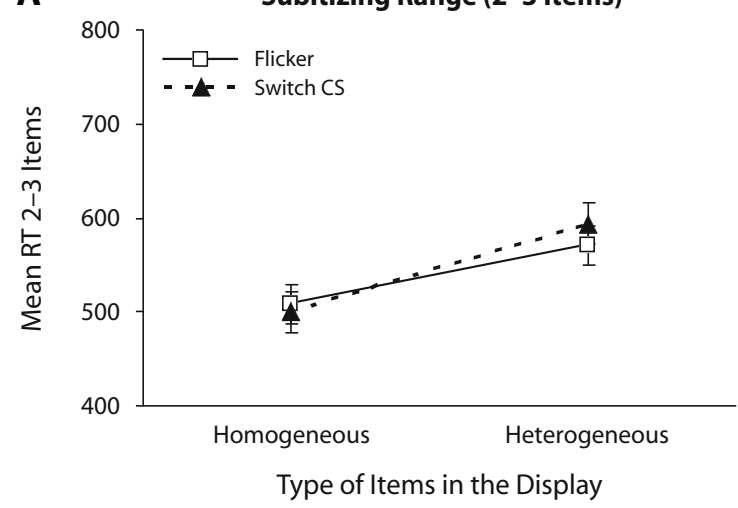

B Counting Range (6-8 Items)

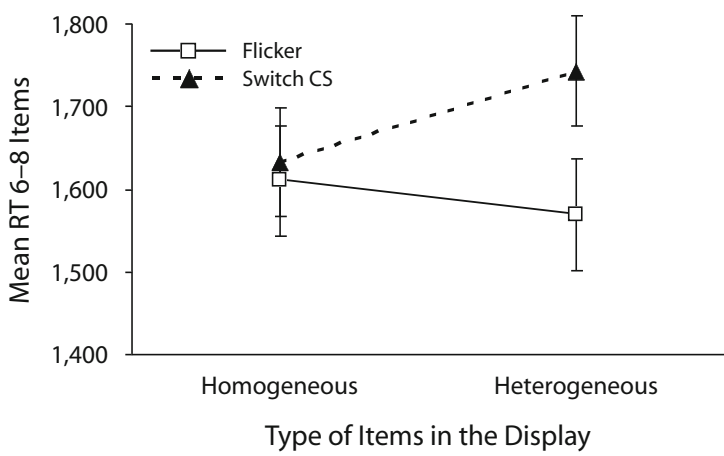

Figure 6. Comparison of the effects of display type and item change in the subitizing range (averaging across latencies for 2-3 items) and the counting range (averaging across latencies for 6-8 items) in Experiments $3 \mathrm{~A}$ and $3 \mathrm{C}$. Figure 6A contains mean latencies for the 2-3 item range. Figure $6 B$ contains mean latencies for the 6-8 item range. Measurements are in milliseconds. Standard error bars are included.

These studies involved manipulations that affected whether participants would adopt an attentional set to select items by type with heterogeneous displays. Results for the six experiments varied dramatically, depending on the number of items and the way in which items changed (as summarized in Table 3).

Because heterogeneity has its strongest effects in the counting range (6-8 items), discussion will begin there. According to Trick and Pylyshyn (1994), group-and-add counting requires selecting first one group of items, then the next, because there are not enough FINSTs to individuate all of the items at the same time. In particular, group-and-add counting requires grouping items, then using attentional selection to choose which group will be enumerated first. Normally, when items have heterogeneous features and participants have not built up expectations about the relative number of each type, participants select on the basis of the location of the items (e.g., moving left to right, top to bottom, in the display), and for homogeneous displays, that is indeed the only way items can be selected. When participants have no reason to use heterogeneity, featural heterogeneity can impede enu- meration, as shown in Experiment 2, perhaps because it provides another grouping cue that competes with grouping by proximity, which is normally used in group-andadd counting (van Oeffelen \& Vos, 1982, 1983, 1984). However, when there was an advantage to selecting items by type - insofar as there was a regularity that could be used to reduce the uncertainty about the addends in groupand-add counting - it sped enumeration substantially in displays of 6-8 items (Experiments 1A and 1B). Thus, heterogeneity could either impede or facilitate counting, depending on whether selection by type could be used to divide the display in half. The magnitudes of the benefits in the counting range were not as large when items flickered, though (Experiments $3 \mathrm{~A}$ and $3 \mathrm{C}$ ).

If items are being selected by type in group-and-add counting for heterogeneous displays, changes in item features should interfere. The results of Experiments 3B and $3 \mathrm{C}$ support this prediction. In fact, the maximal amount of interference occurred when items changed features on two dimensions, although it seemed to make little difference whether items traded features (e.g., the red squares turned into blue triangles and vice versa) or took on completely new features (e.g., red squares turned into green stars, and the blue triangles turned into mauve circles).

In contrast, when items were homogeneous, featural change had no impact on the enumeration of 6-8 items (Experiment 3A). Even though enumerating this many items always requires selecting one group of items, then the next, location-based selection is the only alternative when items are homogeneous, and consequently feature change had no effect. This shows that selection does not necessarily entail that changes in item properties are processed or registered, a finding that may be relevant to the change detection literature.

Within the subitizing range (1-3 items), the pattern of results was very different. Normally, subitizing involves assigning reference tokens (FINSTs) and accessing a number name. Given that FINSTs do not have information about the properties of individuated items, it is small wonder that differences in item features had no effect on enumeration in Experiment 2, where participants were not biased to select items on the basis of their features. Similarly, because items can be individuated all at once (within the first frame of animation in the display) when there are only 1-3 items, it makes sense that changes in item features did not affect enumeration in the subitizing range (Experiments 3A-3C).

However, an attentional set to select items by type impedes enumeration in the subitizing range, because restricting selection first to one type of item and then to the next would only serve to slow the process if there were more than enough reference tokens to individuate all items at once (Experiments 1A and 1B, and the crossstudy comparison of Experiments $3 \mathrm{~A}$ and $3 \mathrm{C}$ ). There are good reasons to suspect that this disadvantage is related to attentional selection rather than to grouping, because grouping by similarity requires heterogeneity in the display, and Experiment 1A showed that an expectation for heterogeneous items slowed the enumeration of 1 item when heterogeneity was blocked. In fact, the effect of 
item heterogeneity was not significantly weaker at 1 item (where there was no actual heterogeneity in the display) than it was at 2 and 3 items (where there was).

It is interesting to consider the overall pattern of results in light of a proposal from the visual search literature about how selection by type might work. It has been known for some time that feature search is faster when participants know the specific dimension by which the target differs from distractors (e.g., Found \& Müller, 1996; Treisman, 1988). Similarly, search is faster when targets and distractors differ in terms of the same dimension on successive trials (Found \& Müller, 1996; Kumada, 2001). There is sometimes a small additional benefit if targets have the same color on successive trials (e.g., feature-specific facilitation; Found \& Müller, 1996; Maljkovic \& Nakayama, 1994). One explanation is that prior knowledge causes that dimension or feature to receive additional weighting when activation is sent the master map of item saliency (Wolfe, 1994: guided search). This makes targets easier to find.

Although this selective weighting account was initially developed to explain performance with distractors, it can be extended to simple enumeration tasks, because when there are large numbers of items there are too many to individuate at once. If group-and-add counting is to occur, it is necessary to select some items, individuate them, unselect them, select the next group, etc. Enumerating one type, then the next, would involve weighting one feature in a dimension, so that it activates locations on the master map, individuating those items, and then reweighting, so that the other feature receives more activation on the master map.

If participants could weight features and dimensions selectively in this way, then it might explain the results of the first two experiments and it would also explain why it was more difficult to enumerate heterogeneous items when they changed features in two dimensions (switch $\mathrm{CS}$, novel CS) than one (switch S, switch C). It should be possible to weight the stable dimension when only one dimension changes, but when both change, it should force participants to select items by location. If participants select items by location, the pattern of results should be the same as in Experiment 2: Heterogeneity should slow enumeration slightly in the counting range and have no effect in the subitizing range. That is not quite what happened. Although heterogeneity slowed enumeration in the counting range, it also slowed it in the subitizing range, when items changed color and shape.

There are two possible explanations. The first is that participants select by location in the switch CS condition (hence the heterogeneity disadvantage in the 6-8 item range), but find it difficult to ignore feature differences between items when features are constantly changing (which produces the heterogeneity disadvantage in the subitizing range). Alternatively, perhaps participants continue to select items by type in heterogeneous displays when items trade features, even when it is so difficult to do that it impedes both subitizing and counting. However, it would be hard to explain how this could work, if selection by type is accomplished by some sort of feature weighting mechanism.

Nonetheless, overall these experiments show that the effects of heterogeneity vary depending on participant ex- pectations and the way that items change. These manipulations have different and sometimes opposite effects on subitizing and counting, which suggests that subitizing and counting must involve different operations. It is important to note that these experiments are at best a partial picture, and other investigations will no doubt reveal complications. For example, these experiments tested only a limited range of numerosities, and when there are larger numbers of items of two types (approximately half of one type, half of the other), participants will have to group items by proximity, even if they continue selecting by type, because there will be more items of each type than can be subitized. Under these circumstances, an attentional set to select by type may take longer to develop, if it develops at all, and the benefits of selection by type may not be as apparent, because the addition will not necessarily be the same every time for every display of a given numerosity. Under these circumstances, an attentional set to select by type might even have a negative impact on accuracy, insofar as participants may adopt the strategy of counting one type of item, then trying to estimate whether there is exactly the same number of the other type, as opposed to one less or one more.

Second, items can differ from one another in a variety of ways, and some types of heterogeneity may affect enumeration differently than others. Trick and Pylyshyn (1994, Experiment 1) found that heterogeneity in item size had no effect on enumeration latencies $(F<1)$. Moreover, heterogeneity may have different effects in enumeration tasks where there is an imposed seriality, such as enumerating words while reading a sentence.

Finally, heterogeneity may have different effects on different operations within spatial enumeration. Heterogeneity can affect grouping operations (see Puts \& de Weert, 1997), and it can facilitate marking, because it provides visible landmarks in the image that can help distinguish enumerated from yet-to-be-enumerated items (Frick, 1987). It is easy to understand why previous investigations produced discrepant results, given the variety of operations necessary for enumeration (e.g., Beckwith \& Restle, 1966; Frick, 1987; Mozer, 1989; Puts \& de Weert, 1997), but this experiment is the first to show how heterogeneity can affect attentional selection.

\section{Implications for the Study of Enumeration}

This article began with a question about whether subitizing exists - or, more properly, whether different operations are used to enumerate small and large numbers of items in situations where discrepancies in RT slope between small and large numbers of items are evident. The results of these experiments suggest that subitizing and counting do involve different operations, because when subitizing and counting are evident there is a manipulation that produces opposite effects on subitizing and counting. This is not to say that subitizing and counting are unrelated; in fact, according to Trick and Pylyshyn (1994), subitizing is part of the groupand-add counting process. There are brain areas that are specific to subitizing or counting and others that the two processes share (e.g., Nan, Knösche, \& Luo, 2006). Nonetheless, counting requires some operations that subitizing does not; as a result, there are dissociations, cases where 
manipulations have different effects on the enumeration of small and large numbers of items. In fact, there many such dissociations (e.g., Dehaene \& Cohen, 1994; Kumada, 2001; Trick, 2005; Trick, Audet, \& Dales, 2003; Trick \& Enns, 1997a, 1997b; Trick \& Pylyshyn, 1994; Tuholski, Engle, \& Baylis, 2001), but this is the first time a single manipulation has produced opposite effects on subitizing and counting. Experiments 1A, 1B, 3A, and 3C showed that when the situation encouraged participants to select by type, there was a crossover interaction, such that a manipulation that speeded counting slowed subitizing. Although discrepancies in RT slope were the initial clue that different processes might be involved in subitizing and counting, ultimately, dissociations are better evidence.

That being said, it is important to note that the accurate enumeration of three or four items is not, by definition, subitizing, and that there are reasons to suspect that subitizing may not occur in every situation. There are a variety of ways to arrive at a numeric response: subitizing; counting; estimating; guessing, and inferring number from cues such as item configuration, contour density, total area occupied by items. As a result, the only way to know which process is being used is to measure both enumeration speed and accuracy. Because the primary evidence for subitizing is discrepancies in RT slope between small and large numbers of items in accurate enumeration, it is difficult to investigate subitizing when RT data are impossible to acquire, as occurs in event enumeration tasks where a sequence unfolds over time at one location and there is no clear place for timing to start.

Nonetheless, it is often assumed that enumeration of a given number of items must always be the same, regardless of task. This assumption manifests itself in several ways. First, it has led some to criticize theories of spatial enumeration because they do not encompass event enumeration (e.g., Wynn, 1996, p. 164). Second, it reveals itself in studies where conclusions are made about the nature of number representations based on a single enumeration task, without replicating the results in tasks where different types of item are enumerated (Cordes, Gelman, Gallistel, $\&$ Whalen, 2001). Finally, this assumption is evident when researchers conclude that subitizing is not a real phenomenon when predicted discrepancies do not occur with every enumeration task (e.g., Cordes \& Gelman, 2005).

Although it is often assumed that all types of enumeration involve the same operations, this assumption is especially questionable given that different dependent variables are used to measure performance in spatial and event enumeration tasks. At best, one could look for manipulations that have different effects on spatial and event enumeration. An examination of past literature shows there are such dissociations. For example, when individuals enumerate sequential light flashes, articulatory suppression interferes even when there are fewer than three flashes of light, although a comparable finger-tapping task has no effect (Logie \& Baddeley, 1987, Figure 4). Conversely, articulatory suppression has little effect on the enumeration of 1-3 dots (ibid., Figures 1 and 2), though a comparable fingertapping task interferes (Trick, 2005). Short-term memory seems to play a more important role in the enumeration of sequential events, as would be expected given that there is no way for sequences that emerge over time to be taken in simultaneously, and given that there is no way to reverse time if an event in a sequence is missed. In contrast, if all of the items are there at once, it may be possible to take in more than one at a time. Moreover, in typical visual-spatial displays it is also possible to look again if an item is missed. Similarly, homogeneity seems to impede the individuation of event sequences, as shown by repetition blindness for repeated tokens of the same type in rapid serial visual presentation (Kanwisher, 1987), a phenomenon that may be related to the attentional blink (Bowman \& Wyble, 2007; Morris \& Harris, 2004).

Not only are there reasons to suspect that subitizing may not occur for sequences of events, there are also times when subitizing does not seem to occur for spatial items. For example, people do not seem to use the fast and accurate subitizing process to enumerate three concentric rectangles, and as a result, the RT slope for enumerating 1-3 concentric rectangles is about the same as the RT slope for enumerating 5-7 concentric rectangles (Trick \& Pylyshyn, 1993, Experiment 1). Similarly, Watson, Maylor, and Bruce (2005b) found that the RT slope when participants were required to enumerate dots that each had a different color was very different from the RT slope required to enumerate the colors of the dots when the number of colors was unrelated to the number of dots (46 msec/item for enumerating 1-3 colored dots as compared with $690 \mathrm{msec} / \mathrm{color}$ for enumerating 1-3 colors). Subitizing only seems to occur with some types of spatial unit, though it may not be restricted to visual-spatial units (Riggs et al., 2006).

In conclusion, enumeration is not always the same, not even the enumeration of three or four. It is undeniable that some form of number representation must be accessed whenever enumeration is performed, but a complete theory of enumeration must also explain unit formation, selection, and individuation. We can enumerate many different things: dots; finger taps; light flashes; ideas in an essay; disappointments in the last month; and so on. There is little reason to expect that the processes used for defining and individuating dots are going to work in exactly the same way as the ones used for disappointments. (When does a disappointment start and end? How do you distinguish one large disappointment from two small ones?) The assumption that all forms of enumeration are the same, regardless of what is being enumerated, has caused all sorts of confusion in the enumeration literature. It is the idea that the phenomena of subitizing and counting necessarily say something about the nature of number representation that needs to be questioned, not the existence of subitizing. There is nothing magical about the number four, but subitizing is more than mere superstition, insofar as there is evidence that variables have different, and sometimes opposite, effects on spatial enumeration performance, when there is evidence of subitizing and counting from RT data. In particular, when a single manipulation produces opposite effects on subitizing and counting, it is time to finally concede that (sometimes) subitizing really occurs. 


\section{AUTHOR NOTE}

This research was funded by a grant from the Natural Sciences and Engineering Research Council of Canada. Parts of this research were presented in a poster session at the annual meeting of the Vision Sciences Society, May 5-10, 2006, Sarasota, FL. I thank Stephanie Gee, Andrea Javor, and Tahlia Perl for their help in the collection of the data Correspondence concerning this article should be addressed to L. M. Trick, Department of Psychology, University of Guelph, Guelph, ON, N1G 2W1 Canada (e-mail: 1trick@uoguelph.ca).

\section{REFERENCES}

AKIn, O., \& Chase, W. (1978). Quantification of three-dimensional structures. Journal of Experimental Psychology: Human Perception \& Performance, 4, 397-410.

Balakrishnan, J. D., \& Ashby, F. G. (1992). Subitizing: Magical numbers or mere superstition? Psychological Research, 54, 80-90.

BeCK, J. (1982). Textural segmentation. In J. Beck (Ed.), Organization and representation in perception. Hillsdale, $\mathrm{NJ}$ : Erlbaum.

Beckwith, M., \& Restle, F. (1966). Process of enumeration. Psychological Review, 73, 437-444.

BoWMAN, H., \& WyBLE, B. (2007). The simultaneous type, serial token model of temporal attention and working memory. Psychological Review, 114, 38-70.

Cordes, S., \& Gelman, R. (2005). The young numerical mind: When does it count? In J. I. D. Campbell (Ed.), Handbook of mathematical cognition (pp. 127-142). New York: Psychology Press.

Cordes, S., Gelman, R., Gallistel, C. R., \& Whalen, J. (2001). Variability signatures distinguish verbal from nonverbal counting for both large and small numbers. Psychonomic Bulletin \& Review, 8, 698-707.

Dehaene, S., \& Cohen, L. (1994). Dissociable mechanisms of subitizing and counting: Neuropsychological evidence from simultanagnosic patients. Journal of Experimental Psychology: Human Perception \& Performance, 20, 958-975.

Found, A., \& Müller, H. J. (1996). Searching for unknown feature targets on more than one dimension: Investigating a "dimensionweighting" account. Perception \& Psychophysics, 58, 88-101.

FrICK, R. W. (1987). The homogeneity effect in counting. Perception \& Psychophysics, 41, 8-16.

Intriligator, J., \& CAVANAGH, P. (2001). The spatial resolution of visual attention. Cognitive Psychology, 43, 171-216.

Jensen, E. M., Reese, E. P., \& Reese, T. W. (1950). The subitizing and counting of visually presented fields of dots. Journal of Psychology: Interdisciplinary \& Applied, 30, 363-392.

Jevons, W. S. (1871). The power of numerical discrimination. Nature, 3, 281-282.

JuLESZ, B. (1984). Toward an axiomatic theory of preattentive vision. In G. M. Edelman, W. E. Gall, \& W. M. Cowan (Eds.), Dynamic aspects of neocortical function (pp. 585-612). New York: Wiley.

KANWISHER, N. G. (1987). Repetition blindness: Type recognition without token individuation. Cognition, 27, 117-143.

Kaufman, E. L., Lord, M. W., Reese, T. W., \& Volkmann, J. (1949). The discrimination of visual number. American Journal of Psychology, 62, 498-525.

KIRK, R. E. (1982). Experimental design: Procedures for the behavioral sciences (2nd ed.). Monterey, CA: Brooks/Cole.

Klahr, D., \& Wallace, J. G. (1976). Cognitive development: An information-processing view. Hillsdale, NJ: Erlbaum.

Kumada, T. (2001). Feature-based control of attention: Evidence for two forms of dimension weighting. Perception \& Psychophysics, 63, 698-708.

Logie, R. H., \& BADDEley, A. D. (1987). Cognitive processes in counting. Journal of Experimental Psychology: Learning, Memory, \& Cognition, 13, 310-326.

Maljkovic, V., \& Nakayama, K. (1994). Priming of pop-out: I. Role of features. Memory \& Cognition, 22, 657-672.

Mandler, G., \& Shebo, B. J. (1982). Subitizing: An analysis of its component processes. Journal of Experimental Psychology: General, 111, $1-22$
Morris, A. L., \& Harris, C. L. (2004). Repetition blindness: Out of sight or out of mind? Journal of Experimental Psychology: Human Perception \& Performance, 30, 913-922.

Mozer, M. C. (1989). Types and tokens in visual letter perception. Journal of Experimental Psychology: Human Perception \& Performance, 15, 287-303.

NAN, Y., KNÖsche, T. R., \& LuO, Y.-J. (2006). Counting in everyday life: Discrimination and enumeration. Neuropsychologia, 44, 1103-1113.

Parkman, J. M., \& Groen, G. J. (1971). Temporal aspects of simple addition and comparison. Journal of Experimental Psychology, 89, 335-342.

Puts, M. J. H., \& DE WeERT, C. M. M. (1997). Does colour influence subitization? Acta Psychologica, 97, 71-78.

Pylyshyn, Z. W. (1989). The role of location indexes in spatial perception: A sketch of the FINST spatial-index model. Cognition, 32, 65-97.

Pylyshyn, Z. W. (in press). Perception, representation and the world: The FINST that binds. In D. Dedrick \& L. Trick (Eds.), Computation, cognition, and Pylyshyn. Cambridge, MA: MIT Press.

Riggs, K. J., Ferrand, L., Lancelin, D., Fryziel, L., Dumur, G., \& Simpson, A. (2006). Subitizing in tactile perception. Psychological Science, 17, 271-272.

Smitsman, A. W. (1982). Perception of number. International Journal of Behavioral Development, 5, 1-31.

Treisman, A. [M.] (1988). Features and objects: The Fourteenth Bartlett Memorial Lecture. Quarterly Journal of Experimental Psychology, 40A, 201-237.

Treisman, A. M., \& Gelade, G. (1980). A feature-integration theory of attention. Cognitive Psychology, 12, 97-136.

Trick, L. M. (2005). The role of working memory in spatial enumeration: Patterns of selective interference in subitizing and counting. Psychonomic Bulletin \& Review, 12, 675-681.

Trick, L. M., Audet, D., \& Dales, L. (2003). Age differences in enumerating things that move: Implications for the development of multiple-object tracking. Memory \& Cognition, 31, 1229-1237.

Trick, L. M., \& ENNS, J. T. (1997a). Clusters precede shapes in perceptual organization. Psychological Science, 8, 124-129.

Trick, L. M., \& ENNS, J. T. (1997b). Measuring preattentive processes: When is pop-out not enough? Visual Cognition, 4, 163-198.

Trick, L. M., \& Pylyshyn, Z. W. (1993). What enumeration studies can show us about spatial attention: Evidence for limited capacity preattentive processing. Journal of Experimental Psychology: Human Perception \& Performance, 19, 331-351.

Trick, L. M., \& PyLYShyn, Z. W. (1994). Why are small and large numbers enumerated differently? A limited-capacity preattentive stage in vision. Psychological Review, 101, 80-102.

Tuholski, S. W., Engle, R. W., \& Baylis, G. C. (2001). Individual differences in working memory capacity and enumeration. Memory \& Cognition, 29, 484-492.

VAn Oeffelen, M. P., \& Vos, P. G. (1982). Configurational effects on the enumeration of dots: Counting by groups. Memory \& Cognition, 10, 396-404.

VAN OEFFELEN, M. P., \& Vos, P. G. (1983). An algorithm for pattern description on the level of relative proximity. Pattern Recognition, 16, 341-348.

VAN OefFelen, M. P., \& Vos, P. G. (1984). Enumeration of dots: An eye movement analysis. Memory \& Cognition, 12, 607-612.

WAtson, D. G., \& MAYLOR, E. A. (2006). Effects of color heterogeneity on subitization. Perception \& Psychophysics, 68, 319-326.

Watson, D. G., Maylor, E. A., \& Bruce, L. A. M. (2005a). Search, enumeration, and aging: Eye movement requirements cause ageequivalent performance in enumeration but not in search tasks. Psychology \& Aging, 20, 226-240.

Watson, D. G., MaYlor, E. A., \& Bruce, L. A. M. (2005b). The efficiency of feature-based subitization and counting. Journal of Experimental Psychology: Human Perception \& Performance, 31, 1449-1462.

Wolfe, J. M. (1994). Guided Search 2.0: A revised model of visual search. Psychonomic Bulletin \& Review, 1, 202-238.

WYNN, K. (1996). Infants' individuation and enumeration of actions. Psychological Science, 7, 164-169.

(Manuscript received April 16, 2007; revision accepted for publication December 28, 2007.) 